\title{
Spectroscopic follow-up of FIRBACK-South bright galaxies
}

\author{
J. Patris ${ }^{1,2, \star}$, M. Dennefeld ${ }^{1,2, \star}$, G. Lagache $^{3}$, and H. Dole ${ }^{4}$ \\ 1 Institut d'Astrophysique de Paris (IAP), 98 bis Bd. Arago, 75014 Paris, France \\ 2 Université Pierre et Marie Curie (Paris VI), 4 place Jussieu 75005 Paris, France \\ 3 Institut d'Astrophysique Spatiale, Bât. 121, Université Paris XI, 91405 Orsay Cedex, France \\ ${ }^{4}$ Steward Observatory, University of Arizona, 933 N Cherry Ave Tucson AZ 85721, USA
}

Received 23 September 2002 / Accepted 2 September 2003

\begin{abstract}
We have performed optical spectroscopy of the brightest $170 \mu \mathrm{m}$ sources in the FIRBACK South Marano (FSM) field. The spectroscopic sample is $90 \%$ complete at the $4 \sigma$ level. Analysis of spectra and comparison with spectra of a faint IRAS $(60 \mu \mathrm{m}$ selected) reference sample is conducted simultaneously. The sources in both samples are characterized by a predominance of emission-line spectra and moderate IR luminosities $\left(10^{10.5}<L_{\mathrm{IR}}<10^{12} L_{\odot}\right)$ The fraction of AGN's is low (about 15\%) and the majority of sources are nearby $(z<0.3)$, dusty, star forming galaxies, with moderate star formation rates (a few $10 M_{\odot}$ per year). The infrared emission of the FSM galaxies shows a colder spectral energy distribution than was expected. The galaxies in both samples (IRAS faint sources and FSM) are essentially undistinguishable with the available data, and seem to represent a population of closeby, cold, star forming galaxies rather neglected up to now. Although their contribution to the far-IR background seems to be low, they deserve a more detailed study on their own to assess their importance in the chemical evolution of the local universe. The contribution of fainter, presumably more distant, galaxies to the far-IR background will be discussed when we will have completed the follow-up of the fainter part of this $170 \mu \mathrm{m}$ survey.
\end{abstract}

Key words. galaxies: infrared - galaxies: evolution - cosmology: observations

\section{Introduction}

FIRBACK (Far InfraRed BACKground) is a $170 \mu \mathrm{m}$ survey carried out by ISO and designed to study the nature of the CIB (Cosmic Infrared Background). It comprises a northern field (FIRBACK N1 and N2; thereafter FN1 and FN2) and a southern one (the FIRBACK Marano field, FSM). Dole et al. (2001) have presented the global results of the survey and concluded that a fraction of the CIB can be resolved into individual sources brighter than $135 \mathrm{mJy}$ (the $3 \sigma$ limit of the survey). A preliminary analysis by Puget et al. (1999) proposes that these sources are a mix of preferentially distant galaxies (at typical redshifts of 1 to 2), whose detection is favoured in the far-IR by the negative K-correction, and which would best reproduce the evolution of the number counts (far in excess over those expected from the local FIR population discovered by IRAS), and of a nearby population of very cold galaxies. This lead naturally to attempts to observe these sources in the sub-mm range, and several of them have been detected with SCUBA in the FN1 field (Scott et al. 2000; Sajina et al. 2003). But until recently, only two redshifts were available, in the FN1 field, two optically faint sources $(I=24 \& 22)$ observed with the Keck telescope, giving $z=0.5$ and 0.9 , respectively

\footnotetext{
Send offprint requests to: M. Dennefeld, e-mail: dennefel@iap.fr

^ Visiting astronomer, European Southern Observatory, La Silla, Chile, under program 66.B-0271.
}

(Chapman et al. 2002). These two sources are identified as ULIRG class galaxies, with merger morphologies and relatively cold dust temperatures. Pending more redshifts, Sajina et al. (2003) used their sub-mm data and spectral energy distributions (SED's) to show, but on a statistical basis only, that FN1 sources appear to show a bimodal galaxy distribution, with normal star-forming galaxies at $\mathrm{z} \simeq 0$ and a $z \sim 0.4-0.9$ population of much more luminous objects. The existence of local, cold galaxies is known from the ISOPHOT serendipity survey (Stickel et al. 2000) which detected a cold ( $\leq 20 \mathrm{~K})$ dust component in many galaxies, but their sample is not complete in any sense and cannot be used for a statistical analysis. The FIRBACK sample is therefore the best sample to study the respective importance of these two populations and its systematic optical follow-up remains to be done.

The aim of this paper is to present a first appraisal of the optical counterparts of the FIRBACK population (more specifically the brighter sources in the southern FSM field) and to derive their nature and distance by spectroscopy. Spectra in the optical range can provide, in addition to redshifts, an analysis of the ionizing source, and an estimate of the extinction. In order to compare the FIRBACK objects with well-studied populations of IR-selected galaxies, we observed also, with the same ESO facilities, a series of IRAS very faint sources selected from the sample of Bertin et al. (1997). The IRAS "Bright Galaxy Sample" of Soifer et al. (1987), observed in 
the optical by Kim et al. (1995) and analyzed by Veilleux et al. (1995), will also be used as a reference on bright IR galaxies.

After analyzing all information that can be extracted from the spectra, and comparing these objects with other populations, we come to the conclusion that the Marano brightest objects are part of a very important population of star forming, dusty, closeby galaxies, that has been essentially neglected up to now. The question is still open for the fainter sources of the FSM survey, which are under study with the ESO VLT.

The paper is organized as follow: the next three sections describe the various data sets and how they were reduced, while Sect. 5 presents and discusses the results. The nature of FIRBACK bright galaxies is summarized in Sect. 6 and the conclusions are presented in the last section.

\section{Data}

\subsection{ISO observations}

The FIRBACK survey consists of three separate high galactic latitude regions which have been observed at $170 \mu \mathrm{m}$ using the ISOPHOT instrument (Lemke et al. 1996) on board ISO (Kessler et al. 1996). Two of these areas are in the northern sky (FN1 and FN2), while the one we are interested in throughout this paper is the southern area, the FIRBACK South Marano or FSM field (Dole et al. 2001).

The FSM region is itself composed of four individual fields, summing up about 22 sources detected at $4 \sigma$ and 13 more at a $3 \sigma$ detection level (Dole et al. 2001), for a total area of 0.95 square degree.

From this source list, we selected the 21 brightest objects (FSM_000 to FSM_020) for spectroscopic follow-up, in order to be complete down to the $4 \sigma$ flux limit $(200 \mathrm{mJy}$ at $170 \mu \mathrm{m})$ in a first phase. Three more objects at lower IR fluxes (FSM_026, FSM_035, FSM_039) have been observed also during this run, as a first exploration of the possible nature of fainter sources.

The coordinates and IR fluxes of those objects are presented in Table 1. The $170 \mu \mathrm{m}$ fluxes are from the FSM catalog (Dole et al. 2001) while the IRAS fluxes have been obtained from the SCANPI $^{1}$ procedure.

\subsection{IRAS reference sample}

In addition to the FSM objects, another set of IR selected galaxies was analyzed for comparison. These are objects extracted from the IRAS Very Faint Source catalog, in the fields studied by Bertin et al. (1997) and selected for their very low $60 \mu \mathrm{m}$ fluxes (in fact the faintest IRAS detections outside the north ecliptic pole). The sample observed is not complete in any sense, but contains a random selection essentially in the $110 \mathrm{mJy} \lesssim f_{v}(60 \mu \mathrm{m}) \lesssim 200 \mathrm{mJy}$ flux range (only a few have stronger fluxes). No particular selection has been made, contrary to other samples where only ULIRG objects where searched for. The technique used to identify those sources is similar to the one used for the FSM sources (see Sect. 3) and

\footnotetext{
${ }^{1}$ http://irsa.ipac.caltech.edu/applications/Scanpi/
}

the spectroscopic follow-up has been done with the same telescope and similar instruments as for the FSM sources. The absolute flux accuracy of the reduced optical spectra in this sample is at the $10 \%$ level. The follow-up of this reference sample is not complete yet and the final results will be published later (Dennefeld et al., in prep). Due to their faintness, and to the relative sensitivity of the various IRAS channels, in most cases the $100 \mu \mathrm{m}$ fluxes are not available.

\subsection{Radio data}

A deep continuum $1.4 \mathrm{GHz}$ radio survey (Dole et al., in prep.) of the ISO FIRBACK field has been performed using the Australia Telescope Compact Array.

A total of 102 hours of observations took place between May 14th and 22nd, 1999, using the 6A configuration, at $1432 \mathrm{MHz}$ and with a correlator bandwidth of $128 \mathrm{MHz}$. Twelve pointings cover the ISO field with an uniform sensitivity in the center. Data were reduced and mosaiced using the Miriad package (Sault et al. 1995).

The non-symmetric synthesized beam has a FWHM of approximatively $12^{\prime \prime} \times 10^{\prime \prime}$ (in RA, Dec), resulting in a source position accuracy of at worst 6 "; with a similar observational strategy, Hopkins et al. $(1998,2003)$ and Gruppioni et al. (1999) showed that the astrometry is better than 2 arcsec. The $5 \sigma$ sensitivity is of the order of $0.27 \mathrm{mJy}$. The Miriad task SFind, using the False Discovery Rate method (Hopkins et al. 2002) was used to extract the sources in the mosaiced image.

The position of the radio sources is marked by dark squares on the finding charts (see Fig. 8). Only the positional information has been used, when necessary, for the FSM source identification, as the flux calibration of this radio sample has still to be completed.

\subsection{Optical observations}

The optical follow-up described here consists of long slit spectroscopy of the galaxies in 23 fields, corresponding essentially to the brighter FSM sources. Imaging and photometry of the whole area is also under way, but the final data are not yet available.

The long slit spectra were obtained on the nights 26-29 November 2000 using EFOSC 2 on the $3.6 \mathrm{~m}$ telescope of ESO in La Silla, Chile. For all spectra, a 1.2" slit was used with grism \#13. This setting gives a resolution of $2.77 \AA$ per pixel and $20 \AA$ per slit width, with a wavelength coverage from $3680 \AA$ to $9340 \AA$. It allows the identification of many features in the spectra of low $z$ galaxies: in particular the $[\mathrm{OII}]_{3726-3729}$ emission lines should be detectable for galaxies in the redshift range $z=0-1.5$, while the $\mathrm{H} \alpha$ Balmer line remains in the range for galaxies closer than $z=0.4$. The spectral resolution is sufficient to separate $\mathrm{H} \alpha$ from $[\mathrm{NII}]_{6583}$ with the help of fitting procedures.

The standard procedure with EFOSC2 is to first take an image of the field, and then select interactively the object(s) to be put in the slit. Rotation of the slit allows to obtain at least two 
Table 1. List of observed FIRBACK South Marano infrared sources.

\begin{tabular}{|c|c|c|c|c|c|}
\hline \multirow[t]{2}{*}{ Source name } & \multirow[t]{2}{*}{$\overline{\alpha \alpha 2000}$} & \multirow[t]{2}{*}{$\overline{\delta 2000}$} & \multicolumn{3}{|c|}{ Infrared flux (mJy) } \\
\hline & & & ISO $170 \mu \mathrm{m}$ & IRAS $100 \mu \mathrm{m}$ & IRAS $60 \mu \mathrm{m}$ \\
\hline FSM_000 & $3^{\mathrm{h}} 09^{\mathrm{m}} 25^{\mathrm{s}}$ & $-54^{\circ} 52^{\prime} 04^{\prime \prime}$ & 497 & 570 & 260 \\
\hline FSM_001 & $3^{\mathrm{h}} 12^{\mathrm{m}} 07^{\mathrm{s}}$ & $-55^{\circ} 17^{\prime} 09^{\prime \prime}$ & 443 & 670 & 590 \\
\hline FSM_002 & $3^{\mathrm{h}} 12^{\mathrm{m}} 29^{\mathrm{s}}$ & $-55^{\circ} 16^{\prime} 30^{\prime \prime}$ & 420 & 310 & 170 \\
\hline FSM_003 & $3^{\mathrm{h}} 11^{\mathrm{m}} 59^{\mathrm{s}}$ & $-55^{\circ} 14^{\prime} 20^{\prime \prime}$ & 369 & $<219$ & $<93$ \\
\hline FSM_004 & $3^{\mathrm{h}} 08^{\mathrm{m}} 37^{\mathrm{s}}$ & $-55^{\circ} 20^{\prime} 45^{\prime \prime}$ & 365 & 430 & 200 \\
\hline FSM_005 & $3^{\mathrm{h}} 10^{\mathrm{m}} 22^{\mathrm{s}}$ & $-54^{\circ} 31^{\prime} 55^{\prime \prime}$ & 301 & $<354$ & $<81$ \\
\hline FSM_006 & $3^{\mathrm{h}} 10^{\mathrm{m}} 45^{\mathrm{s}}$ & $-54^{\circ} 32^{\prime} 05^{\prime \prime}$ & 300 & $<186$ & 210 \\
\hline FSM_007 & $3^{\mathrm{h}} 12^{\mathrm{m}} 10^{\mathrm{s}}$ & $-55^{\circ} 09^{\prime} 00^{\prime \prime}$ & 296 & 260 & 120 \\
\hline FSM_008 & $3^{\mathrm{h}} 12^{\mathrm{m}} 33^{\mathrm{s}}$ & $-54^{\circ} 57^{\prime} 00^{\prime \prime}$ & 269 & $<264$ & $<120$ \\
\hline FSM_009 & $3^{\mathrm{h}} 08^{\mathrm{m}} 42^{\mathrm{s}}$ & $-54^{\circ} 27^{\prime} 28^{\prime \prime}$ & 267 & 370 & $<90$ \\
\hline FSM_010 & $3^{\mathrm{h}} 10^{\mathrm{m}} 16^{\mathrm{s}}$ & $-55^{\circ} 01^{\prime} 37^{\prime \prime}$ & 261 & $<483$ & $<90$ \\
\hline FSM_011 & $3^{\mathrm{h}} 12^{\mathrm{m}} 53^{\mathrm{s}}$ & $-55^{\circ} 09^{\prime} 28^{\prime \prime}$ & 239 & $<198$ & $<78$ \\
\hline FSM_012 & $3^{\mathrm{h}} 08^{\mathrm{m}} 03^{\mathrm{s}}$ & $-54^{\circ} 34^{\prime} 33^{\prime \prime}$ & 232 & $<306$ & $<72$ \\
\hline FSM_013 & $3^{\mathrm{h}} 15^{\mathrm{m}} 18^{\mathrm{s}}$ & $-55^{\circ} 01^{\prime} 26^{\prime \prime}$ & 228 & $<267$ & $<54$ \\
\hline FSM_014 & $3^{\mathrm{h}} 14^{\mathrm{m}} 50^{\mathrm{s}}$ & $-54^{\circ} 59^{\prime} 09^{\prime \prime}$ & 226 & $<159$ & $<75$ \\
\hline FSM_015 & $3^{\mathrm{h}} 10^{\mathrm{m}} 37^{\mathrm{s}}$ & $-54^{\circ} 26^{\prime} 16^{\prime \prime}$ & 225 & 380 & $<105$ \\
\hline FSM_016 & $3^{\mathrm{h}} 13^{\mathrm{m}} 07^{\mathrm{s}}$ & $-54^{\circ} 49^{\prime} 40^{\prime \prime}$ & 214 & 230 & $<72$ \\
\hline FSM_017 & $3^{\mathrm{h}} 08^{\mathrm{m}} 24^{\mathrm{s}}$ & $-54^{\circ} 28^{\prime} 04^{\prime \prime}$ & 210 & - & - \\
\hline FSM_018 & $3^{\mathrm{h}} 10^{\mathrm{m}} 01^{\mathrm{s}}$ & $-55^{\circ} 11^{\prime} 45^{\prime \prime}$ & 207 & $<183$ & $<57$ \\
\hline FSM_019 & $3^{\mathrm{h}} 07^{\mathrm{m}} 28^{\mathrm{s}}$ & $-55^{\circ} 09^{\prime} 07^{\prime \prime}$ & 202 & - & - \\
\hline FSM_020 & $3^{\mathrm{h}} 09^{\mathrm{m}} 31^{\mathrm{s}}$ & $-55^{\circ} 25^{\prime} 04^{\prime \prime}$ & 200 & - & - \\
\hline CFSM_026 & $3^{\mathrm{h}} 09^{\mathrm{m}} 43^{\mathrm{s}}$ & $-54^{\circ} 43^{\prime} 08^{\prime \prime}$ & 160 & - & - \\
\hline CFSM_035 & $3^{\mathrm{h}} 08^{\mathrm{m}} 09^{\mathrm{s}}$ & $-55^{\circ} 09^{\prime} 07^{\prime \prime}$ & 142 & - & - \\
\hline CFSM_039 & $3^{\mathrm{h}} 10^{\mathrm{m}} 22^{\mathrm{s}}$ & $-54^{\circ} 13^{\prime} 05^{\prime \prime}$ & 134 & - & - \\
\hline
\end{tabular}

spectra in a single exposure if necessary. The acquisition image was obtained with a red Bessel filter (ESO number 642), with an exposure time of at least one minute, to allow easy source identification and aperture correction if necessary.

The seeing was generally better than $1^{\prime \prime}$ during this observing run and all the nights were photometric.

\subsection{Reduction procedure}

The spectra were reduced and calibrated using ESO's MIDAS software in the interactive context "LONG". Wavelength calibration was performed using helium argon lamps spectra, and fitting a three degree polynomial. The error on $\lambda$ was found to be less than $3 \AA$, essentially independent of the telescope position.

All the spectra were flux calibrated, after correction of atmospheric extinction, using three spectrophotometric standard stars: LTT 3218, LTT 3864 and LTT 1020 (Hamuy et al. 1992, 1994). The variation between the response curves (two observations for LTT 1020, one for LTT 3864 and one for LTT 3218) was found to be about $2 \%$ and we used an averaged response to calibrate all scientific spectra: we can thus estimate the error on absolute fluxes (apart from slit losses) to be less than 5\%.

\subsection{Analysis of the spectra}

For each calibrated spectrum, the following procedure was employed, making use of ESO/MIDAS routines.

First, a noise spectrum is estimated by summing up the two first scales of a wavelet transform (automatic number of scales of 10 for a 2000 pixels spectrum). The two first scales are indeed below the resolution of the instrument $(20 \AA)$ and thus can be supposed to be pure noise component. This noise spectrum is used: i) to estimate the global $\mathrm{S} / \mathrm{N}$ ratio of the spectrum and ii) to extract the high-frequency noise of the whole spectrum and thus make it more readable.

Then the cleaned spectrum is visually examined, and a hypothesis on $z$ is made. The hypothesis is tested automatically by searching for emission and absorption lines. If more than three separate features are found with a $\Delta z$ lower than 0.001 , the redshift is considered a good guess. The program then searches for all lines of a given list. Each line found is fitted with a deblend MIDAS routine, with a continuum interpolated between two "flat" regions (pre-determined, the same for all objects) around the line. The total flux, equivalent width and measured redshift are given for each line. The final $z$ is determined by averaging over all detected features, and the error is given by the dispersion - it is generally of the order of $5 \times 10^{-4}$ for an average spectrum, and never worse than a few $10^{-3}$.

A rough spectral classification (reported in Col. 8 of Table 2) is done according to the following rules:

- $a$ when no emission line has been detected, so that the only measurable features are the $\mathrm{H}$ and K Ca II lines (at $3933 \AA$ and $3968 \AA$ ) and possibly other absorption lines;

$-e+a$ when emission lines have been detected ( $\mathrm{H} \beta$ equivalent width greater than one $\AA$, other non-Balmer emission lines), along with absorption features;

- agn when high excitation forbidden lines indicate an active nucleus. 
Table 2. Flux measurements for the FIRBACK South Marano sample.

\begin{tabular}{|c|c|c|c|c|c|c|c|c|c|c|c|c|c|c|}
\hline \multirow{2}{*}{$\begin{array}{l}\text { IR source } \\
\text { (1) }\end{array}$} & \multirow{2}{*}{$\begin{array}{l}\text { LIR } \\
(2)\end{array}$} & \multirow{2}{*}{$\begin{array}{l}\text { ID } \\
(3)\end{array}$} & \multirow{2}{*}{$\begin{array}{c}z \\
(4) \\
\end{array}$} & \multirow{2}{*}{$\begin{array}{l}A v \\
(5)\end{array}$} & \multicolumn{2}{|c|}{$[\mathrm{OII}]_{3727}$} & \multirow{2}{*}{$\begin{array}{l}\mathrm{H} \beta \\
(6)\end{array}$} & \multirow{2}{*}{$\begin{array}{c}\mathrm{OIII}]_{4959} \\
(6)\end{array}$} & \multirow{2}{*}{$\begin{array}{c}\mathrm{OIII}]_{5007} \\
(6)\end{array}$} & \multicolumn{2}{|c|}{$\mathrm{H} \alpha$} & \multirow{2}{*}{$\begin{array}{c}{[\mathrm{NII}]_{6583}} \\
(6)\end{array}$} & \multirow{2}{*}{$\begin{array}{l}\text { Nature } \\
\text { (8) }\end{array}$} & \multirow{2}{*}{$\begin{array}{c}\text { Comments } \\
(9)\end{array}$} \\
\hline & & & & & (6) & (7) & & & & (6) & (7) & & & \\
\hline \multirow[t]{2}{*}{ FSM_000 } & 10.9 & $1 *$ & 0.0466 & 3.9 & 1.4 & 256 & 0.70 & - & - & 13 & 234 & 3.6 & $\mathrm{e}+\mathrm{a}$ & $1 \& 2$ interacting \\
\hline & & 2 & 0.0462 & - & - & - & - & - & - & - & - & - & $\mathrm{a}$ & \\
\hline \multirow[t]{2}{*}{ FSM_001 } & 10.6 & $1^{*}$ & 0.0297 & 4.6 & 0.89 & 410 & - & - & 0.14 & 5.6 & 165 & 1.1 & $e+a$ & $1 \& 2$ interacting \\
\hline & & 2 & 0.0289 & - & 0.54 & 26 & - & - & - & - & - & 0.99 & $\mathrm{a}$ & \\
\hline FSM_002 & 10.4 & * & 0.0310 & 4.3 & 0.88 & 269 & - & - & - & 4.2 & 98 & 0.43 & $\mathrm{e}+\mathrm{a}$ & \\
\hline FSM_003 & 11.8 & * & 0.1309 & 7.1 & 0.21 & 2750 & - & - & 0.15 & 1.96 & 360 & 1.1 & $\mathrm{e}+\mathrm{a}$ & LINER ? \\
\hline FSM_004 & 11.3 & * & 0.0782 & 3.4 & 4.9 & 476 & 1.3 & 9.2 & 24 & 11.8 & 147 & 5.6 & agn & \\
\hline \multirow[t]{2}{*}{ FSM_005 } & 9.6 & $1 *$ & 0.1505 & - & 0.52 & 25 & - & 0.21 & 0.30 & 3.9 & 32 & 0.81 & $\mathrm{e}+\mathrm{a}$ & FSM_005 $=1+2$ \\
\hline & & $2^{*}$ & 0.1516 & - & 0.19 & 9.2 & - & 0.22 & - & 1.8 & 15 & - & $e+a$ & $1 \& 2$ merging \\
\hline \multirow[t]{2}{*}{ FSM_006 } & & 1 & 0.0480 & 2.6 & 0.29 & 9.1 & - & 0.071 & 0.13 & 0.28 & 1.8 & - & $\mathrm{e}+\mathrm{a}$ & \\
\hline & 12.1 & $2^{*}$ & 0.2050 & 2.0 & 0.68 & 10 & 0.22 & 0.087 & 0.13 & 3.5 & 15.4 & 0.4 & $\mathrm{e}+\mathrm{a}$ & \\
\hline FSM_007 & 11.2 & $*$ & 0.0923 & 2.6 & 1.5 & 52 & 0.71 & 0.29 & 0.48 & 7.7 & 53 & 2.3 & $\mathrm{e}$ & \\
\hline FSM_008 & 12.2 & * & 0.2282 & 2.1 & 0.34 & 5.4 & 0.18 & 0.063 & 0.23 & 2.2 & 9.9 & 0.92 & $e+a$ & \\
\hline \multirow[t]{2}{*}{ FSM_009 } & 11.9 & $1^{*}$ & 0.1918 & 2.0 & 0.63 & 9.5 & - & 0.067 & 0.18 & 1.9 & 8.3 & 0.45 & $e+a$ & interacting with 2 \\
\hline & & 2 & 0.1915 & 2.1 & 0.23 & 3.9 & - & - & - & 0.56 & 2.6 & - & $e+a$ & \\
\hline \multirow[t]{2}{*}{ FSM_010 } & 12.2 & $1 *$ & 0.2569 & - & - & - & - & - & - & - & - & - & $\mathrm{a}$ & FSM_010 = $1+2$ \\
\hline & & $2 *$ & 0.2578 & 1.5 & 0.25 & 1.9 & 0.082 & 0.019 & 0.051 & 0.72 & 2.2 & 0.30 & $\mathrm{e}+\mathrm{a}$ & $1 \& 2$ interacting? \\
\hline FSM_011 & 12.9 & $*$ & 0.6576 & - & 0.95 & 45 & 0.75 & 1.5 & 4.7 & - & - & - & agn & pointlike \\
\hline \multirow[t]{2}{*}{ FSM_012 } & 12.1 & $1 *$ & 0.1925 & 2.5 & 1.2 & 33 & 0.43 & 0.12 & 0.36 & 5.0 & 31 & 1.6 & $\mathrm{e}+\mathrm{a}$ & \\
\hline & & 2 & 0.0547 & 4.1 & 0.79 & 196 & - & - & - & 1.4 & 29 & 0.15 & $\mathrm{e}+\mathrm{a}$ & \\
\hline FSM_013 & & 1 & 0.0546 & 1.8 & 0.40 & 4.6 & - & - & 0.15 & 0.51 & 1.97 & 0.030 & $e+a$ & \\
\hline & 12.2 & $2^{*}$ & 0.2389 & 2.2 & 0.16 & 3.1 & - & - & 0.09 & 0.46 & 2.3 & 0.085 & $\mathrm{e}+\mathrm{a}$ & FSM_013 $=2+3$ \\
\hline & & $3 *$ & 0.2541 & 2.7 & 0.17 & 6.5 & 0.077 & 0.14 & 0.16 & 0.43 & 3.2 & 0.61 & $e+a$ & LINER ? \\
\hline FSM_014 & $11.5(\mathrm{~A})$ & $1 *$ & 0.1579 & - & 0.49 & 23 & 0.092 & 0.071 & 0.16 & 0.27 & 2.3 & - & $e+a$ & IR flux shared $A=1+2$ \\
\hline & & $2^{*}$ & 0.1589 & - & 0.61 & 29 & 0.22 & 0.10 & 0.22 & 0.43 & 3.6 & - & $\mathrm{e}+\mathrm{a}$ & $1 \& 2$ interacting \\
\hline & $11.8(\mathrm{~B})$ & $3 *$ & 0.2915 & 1.6 & 0.23 & 2.1 & - & - & - & 0.48 & 1.6 & 0.28 & $\mathrm{e}+\mathrm{a}$ & IR flux shared $B=3$ \\
\hline FSM_015 & 10.8 & * & 0.0578 & 3.9 & 0.31 & 56 & - & - & - & 0.76 & 13 & 0.12 & $e+a$ & \\
\hline FSM_016 & 11.5 & $*$ & 0.1302 & 3.3 & 0.50 & 43 & 0.53 & 0.079 & 0.15 & 8.3 & 96 & 0.79 & $e+a$ & \\
\hline FSM_017 & 11.0 & $1 *$ & 0.0744 & 5.4 & 0.47 & 685 & - & - & 0.091 & 1.9 & 104 & - & $\mathrm{e}+\mathrm{a}$ & \\
\hline & & 2 & 0.1295 & 2.7 & 0.22 & 8.2 & 0.053 & - & 0.081 & 0.31 & 2.3 & - & $\mathrm{e}+\mathrm{a}$ & \\
\hline & & 3 & 0.2991 & - & 0.17 & 8.2 & 0.089 & - & - & 0.17 & 1.5 & - & $\mathrm{e}+\mathrm{a}$ & \\
\hline FSM_018 & 12.0 & 1 & 0.2329 & - & - & - & - & - & - & - & - & - & $\mathrm{a}$ & \\
\hline & & 2 & 0.2341 & - & - & - & - & - & - & - & - & - & $\mathrm{a}$ & \\
\hline FSM_019 & & 1 & 0.0725 & 2.7 & 0.65 & 26 & 0.084 & 0.14 & 0.084 & 3.6 & 27 & - & $\mathrm{e}+\mathrm{a}$ & \\
\hline & 12.3 & $2 *$ & 0.2568 & 2.2 & 0.13 & 2.7 & - & - & - & 0.75 & 3.9 & - & $\mathrm{e}+\mathrm{a}$ & \\
\hline FSM_020 & 11.0 & $1^{*}$ & 0.0714 & 2.5 & 1.2 & 35 & - & 0.29 & 0.27 & 5.3 & 33 & 0.93 & $\mathrm{e}+\mathrm{a}$ & $1 \& 2$ interacting \\
\hline & & 2 & 0.0713 & 1.3 & 1.5 & 8.7 & 0.32 & 0.35 & 1.0 & 2.0 & 5.2 & - & $e+a$ & \\
\hline CFSM_026 & 10.9 & * & 0.0780 & 4.7 & - & - & - & - & - & 2.0 & 66 & - & $e+a$ & \\
\hline CFSM_035 & 12.5 & * & 0.5902 & - & 0.49 & 23 & 4.1 & - & 1.6 & - & - & - & agn & pointlike \\
\hline CFSM_039 & $10.9(\mathrm{~A})$ & $1 *$ & 0.1413 & 1.3 & 1.4 & 8.0 & 0.38 & 0.36 & 0.88 & 2.8 & 7.2 & 0.67 & $\mathrm{e}+\mathrm{a}$ & IR flux shared $A=1$ \\
\hline & 11.7(B) & $2 *$ & 0.2684 & 2.2 & 0.18 & 3.7 & 0.098 & - & - & 1.2 & 6.5 & 0.44 & $\mathrm{e}+\mathrm{a}$ & IR flux shared B = 2 \\
\hline & $\begin{array}{l}\text { (1) Name } \\
\text { (3) Identif } \\
\text { (5) Extinc } \\
\text { (7) Flux c } \\
\text { (9) Comm }\end{array}$ & $\begin{array}{l}\text { M 1 } \\
\text { on nu } \\
4_{V}=\end{array}$ & $\begin{array}{l}\mathrm{m} \text { publis } \\
\mathrm{r} \text { of the } \\
E(B-\mathrm{I} \\
\text { absorpti } \\
\text { an be att }\end{array}$ & $\begin{array}{l}\text { sourc } \\
\text { al ob }\end{array}$ & $*: \mathrm{Ob}$ & consid & $\begin{array}{l}\text { to be th } \\
\text { 3), with }\end{array}$ & $\begin{array}{l}\text { source. } \\
3.2 \text {. } \\
\text { sect. 3.3). }\end{array}$ & $\begin{array}{l}\text { (2) } \log 10 \text { of } \\
\text { (4) Redshift } \\
\text { (6) Main em } \\
\text { (8) Spectral }\end{array}$ & $\begin{array}{l}1 \text { IR l } \\
\text { sured } \\
\text { on lin } \\
e+\end{array}$ & $\begin{array}{l}\text { osity i } \\
\text { n lines } \\
\text { xes, } 1 \\
\text { ission }\end{array}$ & $\begin{array}{l}\text { (see Sect. } \\
\mathrm{r} \simeq 5 \times 10 \\
\mathrm{erg} / \mathrm{s} / \mathrm{cm} \\
\text { absorptio }\end{array}$ & $\begin{array}{l}\text { ee Sect. } 2 \\
\text { or }<10 \% \text {. } \\
\text { s. } a \text { : no e }\end{array}$ & ion line. \\
\hline
\end{tabular}




\section{Source identification}

Identification of optical counterparts of far IR sources is a complicated process due to the large "beam" size of ISOPHOT. FSM $170 \mu \mathrm{m}$ PHOT sources are ascribed to a 100 " diameter circle, which corresponds to a localising probability of about $93 \%$, as discussed by Dole et al. (2001). The consequence is that usually more than one optical galaxy lies in the error circle, and this multiplicity of course increases when going to fainter optical magnitudes. As we deal here with the brightest PHOT sources only, the problem is however less severe, and we have, whenever possible, taken spectra of all the (bright) galaxies lying within the error circle.

In about half the cases, only one optical galaxy was found (although sometimes more than one spectrum was taken, to check other optical candidates, which then proved to be stars). If more than one optical galaxy is found inside the error circle, we then used the following criteria to pursue the identification further:

- the spectrum should look similar to a "standard" starburst galaxy, i.e. have emission lines (particularly [OII] or $\mathrm{H} \alpha$ ), a reddened continuum, etc.;

- the radio emission in the ATCA data at $1.4 \mathrm{GHz}$ (Dole et al., in preparation), if available in the field, should correspond to this galaxy. The better angular resolution (at the arc-second level) of the radio observations allows in most cases to identify without ambiguity the optical candidate associated with the radio source. This general procedure relies on the assumption that the IR PHOT source is of a similar nature than the standard IRAS galaxies, powered by star-formation or an AGN, and where the well known radio/IR correlation holds over a large range of fluxes. This "bias" would, at this early stage, prevent identification of sources of different nature, should they exist in this sample. However, the "radio emission" criterion was decisive only for a few objects (four IR sources) and we have found no case (in this bright sample) where there was only one radio source, corresponding to a non-emission lines galaxy.

If several optical objects have similar properties (same redshift, emission lines, radio emission) and all lie inside the error circle, we assume they all contribute to the IR emission and thus we sum all the optical contributions. The redshift attributed to the IR object is then the average of the optical redshifts. This is the case for four objects: FSM_005, FSM_010, FSM_013 and FSM_018.

More difficult is the case when two galaxies with different redshifts but otherwise similar properties (emission lines, radio emission...) lie in the error circle. Both candidates probably contribute to the final infrared flux, and, since there is no perfect criterion to chose one candidate or the other, we chose to distribute the IR flux proportionnaly to the $\mathrm{H} \alpha$ line intensity (this assumes a linear relation between IR and emission line fluxes, which holds only roughly). These problematic cases are fortunately rare enough here ( 1 case out of 28 for the IRAS sample, 2 out of 24 for the FSM sample) that they will not affect our general conclusions. We found only one case where none of this criteria could be used: in FSM_018, there is no detected radio emission, and none of the observed galaxies does show emission lines (see discussion of individual sources).

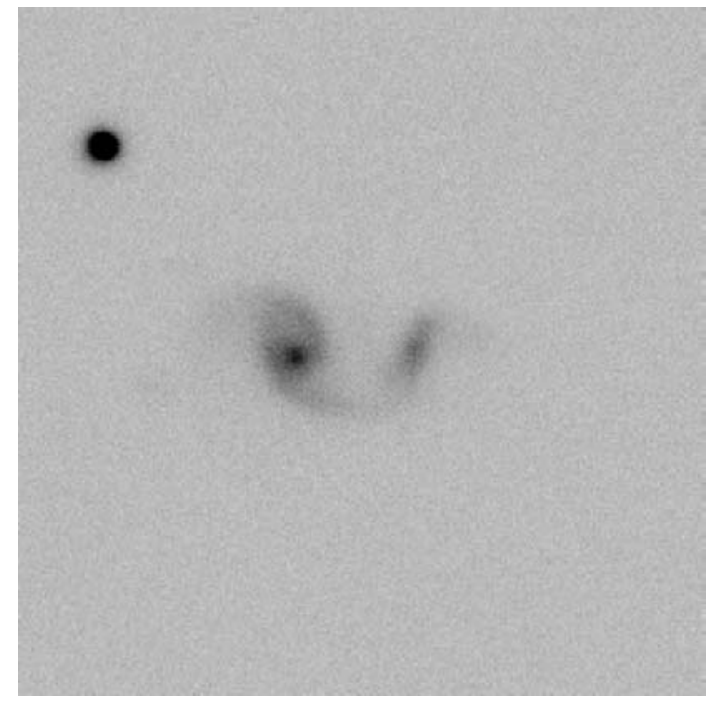

Fig. 1. $R$-band image of FSM_005 infrared source, showing galaxy number 1 (left) \& 2, taken with the ESO $3.6 \mathrm{~m}$ telescope in La Silla. This picture shows clearly the two merging galaxies. The field is rotated (the star is due north of the galaxies) in order to place the slit across both galaxies).

Figure 8 presents the DSS images with a superposed infrared error circle, radio identifications (when available), spectrograph slit position and final identification(s) (the optical galaxy finally associated with the IR emission is indicated by an asterisk, see also Table 2). In the following, we discuss each identification separatly.

\section{Main catalog:}

- FSM_000: Two large galaxies have been placed on the slit. The infrared source is associated with the large galaxy inside the error circle, witch is also a radio source. The second galaxy (also a radio source, but outside the circle) has not a starburst spectrum and is therefore assumed to contribute little or nothing to the IR emission.

- FSM_001: The infrared source is associated with the large galaxy inside the error circle, which is also a radio source, object \#2 being out of the error circle and with no radio emission.

- FSM_002: Only one large galaxy is in the error circle, but there is no radio emission.

- FSM_003: Among the numerous stars of the nearby globular cluster lies a large galaxy, which is also a radio source.

- FSM_004: One large radio bright galaxy.

- FSM_005: On close examination (see Fig. 1), the central optical source is in fact a couple of merging galaxies (of whom one at least is a radio source). We associated the infrared flux with both optical galaxies (and thus added the emission line intensities).

- FSM_006: The galaxy associated with the infrared flux (\#2) is inside the error circle, and is associated with radio emission. A contribution from galaxy \#1 is possible too.

- FSM_007: This large galaxy, close to the globular cluster, is also a radio source.

- FSM_008: One galaxy is in the error circle, which is also a radio source. 
- FSM_009: There are two galaxies but galaxy \#2 is on the border of the error circle, has weak emission lines and no radio counterpart. We thus attribute the IR flux to galaxy \#1 only.

- FSM_010: Two faint galaxies have been observed spectroscopically. One is well inside the error circle but has no radio counterpart, the other is on the limit of the error circle and is associated with a radio source. Since both galaxies are at the same redshift (though separated by about $250 \mathrm{kpc}$ with a standard, $H_{0}=65 \mathrm{~km} / \mathrm{h} / \mathrm{Mpc}$ cosmology) we added the optical fluxes. A much fainter object (not observed spectroscopically) and coinciding with a radio source could contribute also.

- FSM_011: No bright galaxy was visible inside the error circle, so we placed two slits to check various faint optical sources. We found a few stars, two very weak galaxies with no emission line, and a point-like active galaxy with bright emission lines and rather high redshift $(z=0.66)$. The infrared flux was attributed to this object. Note that this object has the highest IR luminosity of the whole sample, by far.

- FSM_012: One of the few cases where radio emission was decisive: galaxy \#1 was chosen because of it (although galaxy \#2 has week emission too).

- FSM_013: Two of the three galaxies in the slit are well inside the error circle, with similar redshifts. They are probably interacting, and thus we considered that both contributed to the infrared flux (optical fluxes were added).

- FSM_014: All galaxies lie within the error circle, with no radio identification available. Without further information available, we divided the infrared flux in two parts, proportionnally to the $\mathrm{H} \alpha$ emission line strength: one part (infrared object FSM_014 A) is attributed to galaxies \#1 and \#2 (two galaxies probably interacting at $z=0.16$, optical fluxes added), and the other part (infrared object FSM_014 B) to galaxy \#3 $(z=0.3)$.

- FSM_015: One large galaxy at the center of the error circle, no radio emission.

- FSM_016: One large galaxy at the error circle's center, with radio emission.

- FSM_017: Two galaxies are inside the error circle, we chose \#1 because it has radio emission.

- FSM_018: We observed spectroscopically both galaxies inside the error circle, however, none of them shows any emission line. There is no radio emission. This would be the only case where the optical counterpart has no emission line. The identification here is still unsecure.

- FSM_019: Only one galaxy lies inside the error circle, has emission lines but no radio emission: we consider it as the IR counterpart. Note however that another faint source lies on the borderline, with radio emission (which was not known at the time of our observations), and could contribute to the IR flux also.

- FSM_020: One large galaxy inside the error circle, with optical emission and radio emission.

\section{Complementary catalog:}

- FSM_026: One large galaxy at the center of the error circle. Weak optical emission but no radio emission.

- FSM_035: From the two radio sources, one seems to be a quiet galaxy (no redshift determined) and the other a bright point-like radio-loud QSO at $z=0.6$. We thus provisionnally attribute the infrared flux to the AGN alone.

- FSM_039: Two galaxies with no radio detection lie in the error circle, \#1 at $z=0.14$, rather bright but off-centered, \#2 at $z=0.27$, fainter, centered. We divided the IR flux proportionnally to the $\mathrm{H} \alpha$ emission line intensity.

\section{Various corrections}

\subsection{Aperture correction}

We have used a $1.2^{\prime \prime}$ slit for the observations, wider than the seeing during the whole run. While for point-like source, we may then consider that most of the object's flux is transmitted, for extended galaxies, on the contrary, a fraction of the flux is lost at the entrance, and should be evaluated. The question of aperture correction is however difficult, and different solutions have been used in the literature:

- correction using photometry: the total spectrophotometric flux can be corrected by comparing it to the photometric flux (magnitude) (solution used for instance in the CFRS, Tresse et al. 1996). However, this procedure assumes a regular distribution of the surface brightness over the galaxy, and when emission lines are concerned (our main interest here) is far from being satisfactory. If (as is probably the case for our objects) the star forming region is essentially circumnuclear, such a correction would grossly overestimate the total emission.

- no correction at all, if the slit is considered wide enough (e.g. in Sullivan et al. 2000 for the FOCA UV-galaxies follow-up). Our case is intermediate between these two extremes, but because the emission is mostly circumnuclear in IRAS galaxies, we decided not to correct the spectra for this effect. In doing this, the measured fluxes are certainly lower limits, but should not greatly underestimate the real values for absolute fluxes. It does not affect, of course, equivalent widths and flux ratios.

\subsection{Underlying stellar absorption lines}

The Balmer emission lines have a particular importance in the spectrum: the "Balmer decrement" is the main indicator of dust reddening (see next section); the diagnostic diagrams use $\mathrm{H} \beta$ and/or $\mathrm{H} \alpha$ with forbidden lines to assess the nature of the ionizing source; $\mathrm{H} \alpha$ is one of the best star formation indicators. Yet, atmospheres of A and B stars, generally present in the underlying stellar populations of galaxies, absorb in the same lines, thus altering the resulting spectrum significantly. It is then of particular importance to correct for this underlying absorption by stars. Several solutions have been applied in the past, depending on the quality of the spectra and the available information:

- A constant correction applied to the equivalent width of the emission line. The value applied is usually around $2 \AA$ for HII regions, and can go up to $5 \AA$ (Kennicutt 1992). This is an average value, not well calibrated on galactic objects. This type of correction is usually used for large samples where individual properties are neglected and when no other solution is available. 

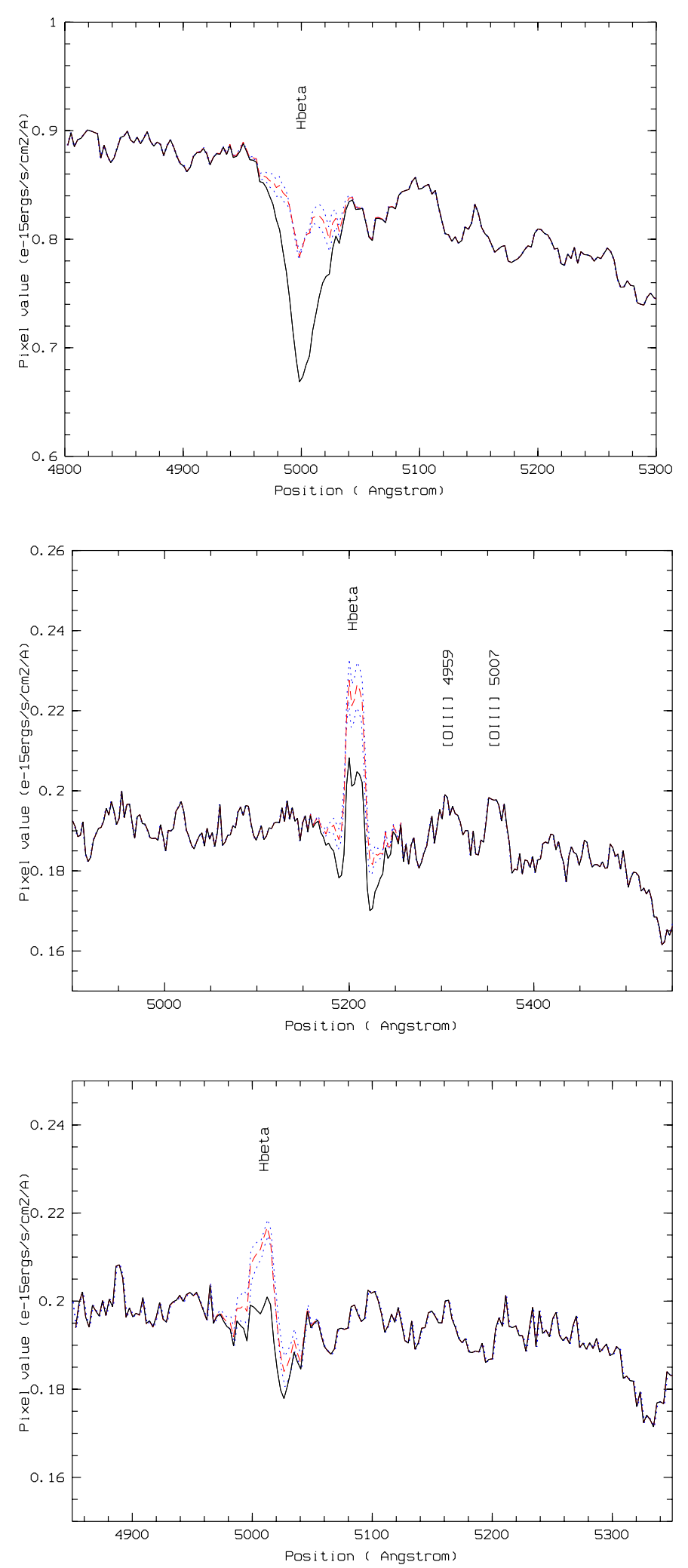

Fig. 2. Correction for stellar absorption. First panel: test, correction of a pure absorption $\mathrm{H} \beta$ feature. The solid line is the flux before correction, the dotted ones are the fluxes corrected by using either $\mathrm{H} \delta$ or $\mathrm{H} \gamma$, the dashed line is the average of both corrections. Either of the lines used for the correction gives similar results and the residuals are at the level of the noise. Second panel: application to a blended line (removal of the absorption line under $\mathrm{H} \beta$ emission). Third panel: an emission line that was too low to be distinguished from the noise (because of the absorption by stars) appears after the correction.
- Fit the wings of the absorption lines. These wings are usually seen on both sides of the emission line. This technique has been used for instance by Sullivan et al. (2000) or Kim et al. (1995) to reconstruct both the absorption and the emission features. However, this method requires a very good spectral resolution and is very sensitive to noise. Fitting a deep absorption line on very faint wings can lead to large errors in the final flux determination. Furthermore, if other emission lines lie close to the Balmer line (like for instance $[\mathrm{NII}]_{6548}$ and $[\mathrm{NII}]_{6583}$ ) the absorption wings will be completely lost, reducing the accuracy of the method.

- Use of the higher order Balmer lines. This is the most efficient method, which makes use of the $\mathrm{H} \delta$ and $\mathrm{H} \gamma$ lines: the emission decreases rapidly with the order of the line whereas the absorption increases. One can compute (e.g. Osterbrock 1989) that the ratio of the $\mathrm{H} \alpha$ and $\mathrm{H} \gamma$ emission lines is (case $\mathrm{B}$ approximation):

$E(\mathrm{H} \alpha) / E(\mathrm{H} \gamma)=6.1$

while from Menzel et al. (1969), we get, for the ratio of the EW of the absorption lines of the same transitions:

$A(\mathrm{H} \gamma) / A(\mathrm{H} \alpha)=1.26$.

We thus have:

$\frac{A b s}{E m}(\mathrm{H} \gamma)=7.7 \times \frac{A b s}{E m}(\mathrm{H} \alpha)$.

This means, as is well known, that most frequently emission is dominant for the $\mathrm{H} \alpha$ line whereas absorption is dominant for the $\mathrm{H} \gamma$ line.

In the FSM field we have enough spectral resolution and wavelength coverage to measure Balmer absorption lines of higher order in all the spectra. In the IRAS sample on the contrary, due to a slightly different instrumental setting (further red), for many spectra only $\mathrm{H} \alpha$ is in the observed range: for those spectra we therefore applied the average global correction obtained for the sample (see below).

In practice, to correct the $\mathrm{H} \beta$ emission line from underlying absorption, we fitted the $\mathrm{H} \delta$ and $\mathrm{H} \gamma$ absorption lines and derived from them the absorption lines at $4961 \AA$ and $6563 \AA$ respectively. The original spectrum is then divided by this absorption feature, reconstructing the "original" emission line. In Fig. 2 we show the efficiency of this method: the first image shows the results of the method on a pure absorption spectrum, and the two others illustrate how the $\mathrm{H} \beta$ line is recovered, even when it was too low (due to the presence of stellar absorption) to be distinguished from the noise.

$\mathrm{H} \gamma$ is usually blended with a large $\mathrm{Mg}$ absorption band, so we prefer to use $\mathrm{H} \delta$ when available. When both lines are clearly detected, we check that the results are consistent (given the error bars) and correct $\mathrm{H} \alpha$ and $\mathrm{H} \beta$ from the average result.

If none of these two lines is detectable in absorption, (or if they are in emission only), we consider that the absorption by stars is negligible and make no correction. In several spectra, the correction makes $\mathrm{H} \beta$ pop out of the noise. When $\mathrm{H} \beta$ doesn't appear even after the correction (whereas $\mathrm{H} \alpha$ is visible in emission) we deduce that $\mathrm{H} \beta$ is completely absorbed by dust, and 
Table 3. Summary of the two observational samples.

\begin{tabular}{lcc}
\hline \hline Sample name & FSM & IRAS \\
\hline Number of IR sources: & & \\
- initial IR photometric sample & 24 & 38 \\
- optical identifications and redshifts & 26 & 28 \\
- with H $\alpha$ emission line & 23 & 21 \\
\hline
\end{tabular}

Comments: three IR sources (two in the FSM sample and one in the IRAS sample) have been split into two optical objects.

we then use the noise in the continuum to estimate an upper value for $\mathrm{H} \beta$.

We find an average correction of $3 \AA$ for the FSM sample and $3.35 \AA$ for the IRAS sample (average over the corrected spectra -not including the non-corrected ones).

\subsection{Extinction}

For the FSM sample, we can neglect the foreground galactic extinction because of the high galactic latitude of the field $\left(b=-52^{\circ}\right)$. In most FSM galaxies, both $\mathrm{H} \alpha$ and $\mathrm{H} \beta$ are available in emission, so we can use the Balmer decrement to estimate the extinction. This method relies on several assumptions: - the intrinsic $(\mathrm{H} \alpha / \mathrm{H} \beta)_{i}$ ratio has to be known. Indeed theoretical calculations have shown that for a case $\mathrm{B}$ recombination this ratio is almost independent of density and temperature. We will use $(\mathrm{H} \alpha / \mathrm{H} \beta)_{i}=2.86$, the value given by Osterbrock (1989) for a density of $100 \mathrm{~cm}^{-1}$ and a temperature of $10000 \mathrm{~K}$.

- the general form of the extinction curve with wavelength is supposed to be known. We used the Howarth (1983) extension of Seaton's (1979) extinction law for the Milky Way. Other extinction curves used in the literature do not differ widely, at least for the optical range of the spectrum.

We can thus compute the amount of absorption in the visible, $A_{V}$, for each object, using $R=3.2$ (Seaton 1979) and $X(\lambda)$ as described in Howarth (1983). The $A_{V}$ value is included in Table 2.

\section{Results}

We try here to characterize the galaxies selected by the $170 \mu \mathrm{m}$ survey, and compare them to the IRAS sample. All luminosities were computed with a standard cosmology: $\Omega_{\Lambda}=0.7, \Omega_{\mathrm{M}}=$ 0.3 and we took a Hubble constant of $H_{0}=65 \mathrm{~km} \mathrm{~s}^{-1} \mathrm{Mpc}^{-1}$. Due to the small distance of the detected objects, the derived parameters are rather insensitive to the adopted cosmology.

The results of the spectral analysis are presented in Table 2 for the main emission lines used in the discussion.

\subsection{Amount of dust}

Figure 3 presents the histograms of $A_{V}$ (after correction from underlying stellar absorption) for both samples, and Table 4 presents the statistics of the distribution for all spectra observed (excluding occasional stars). The reason to use for the statistics all the observed spectra is because when several galaxies are associated to one IR source, an "average" extinction factor would
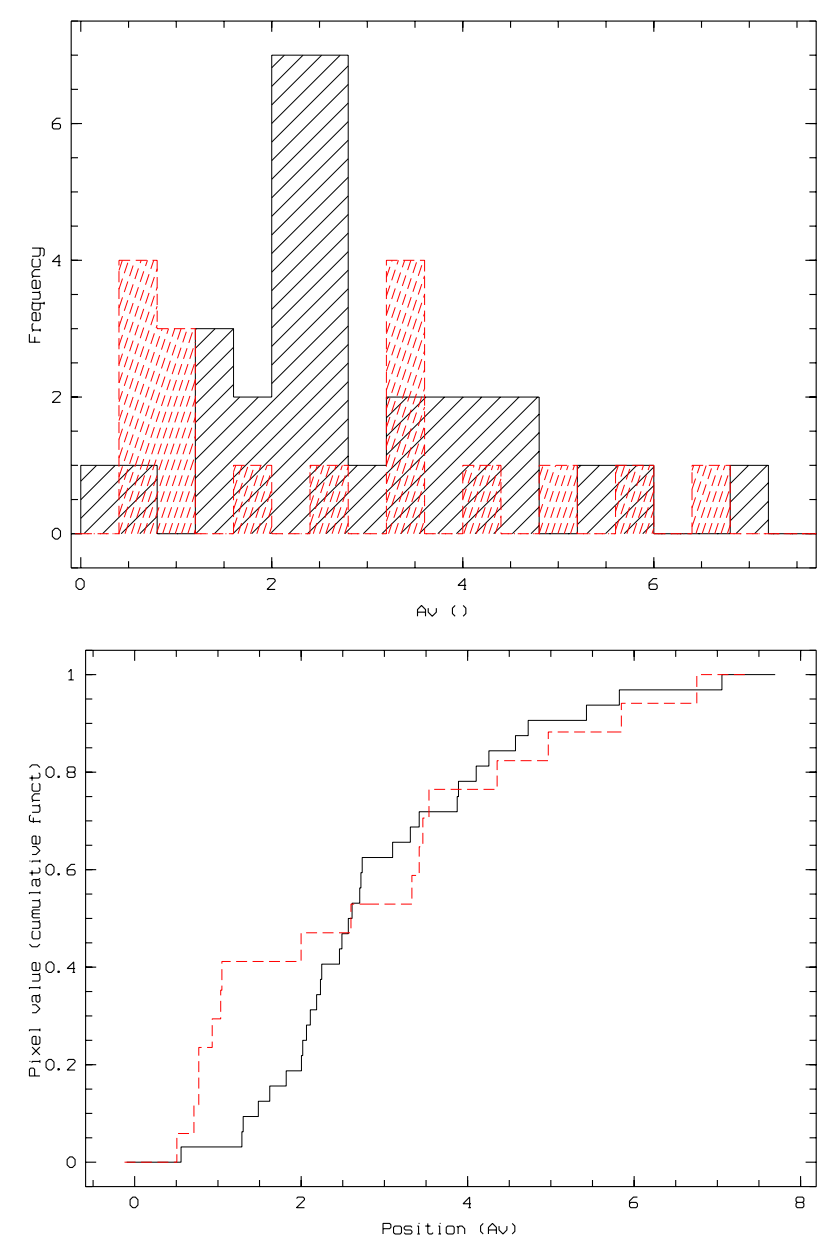

Fig. 3. Distribution of absorption by dust. Top: histograms of the $A_{v}$ values, with median hashing corresponding to FSM galaxies and inclined hashing to the IRAS sample. Bottom: cumulative function of $A_{v}$ for: i) FSM sample (solid curve) and ii) IRAS reference sample (dotted curve).

have no real meaning. The result of a Kolmogorov-Smirnov test on the cumulative functions shows that the two samples have a reasonable chance of coming from the same initial distribution (a $D$ parameter of less than 0.4 , with $n=17$, leads to a $p(D)=25 \%$, that is to say that two samples drawn from the same distribution have $25 \%$ chances of being farther apart than these two samples; see Miller 1956).

We conclude that the two samples are remarquably alike as far as extinction is concerned ( $A_{V} \sim 3$ for both samples). They are also very similar to the "Bright galaxies sample" (Veilleux et al. 1995, thereafter BGS), where $A_{V}$ has an average value of 3.6 (with our adopted value of $R=3.2$ ), but rather far from UV-selected samples $\left(A_{V} \simeq 1\right.$; Sullivan et al. 2000 for the FOCA galaxies). The extinction is also significantly higher than in the CFRS $z<0.3$ sample, where $A_{V}=1.52$ for objects selected in the $I$ band (and is an upper limit, as no correction has been applied there for underlying stellar absorption). This effect is not surprising: it is quite clear that $170 \mu \mathrm{m}$ and $60 \mu \mathrm{m}$ observations both select heavily obscured objects.

We want to stress that an extinction of $A_{V}=2.9 \mathrm{mag}$ (average value) results in a reduction of a factor 9 (or an optical depth of $\tau(\mathrm{H} \alpha)=2$ ) in the $\mathrm{H} \alpha$ line intensity, and a factor 
Table 4. Extinction parameter for individual galaxies in both samples. The two distributions are similar, given the small statistics, and cover the same range.

\begin{tabular}{llllll}
\hline \hline $\begin{array}{l}\text { Sample } \\
\text { name }\end{array}$ & $\begin{array}{l}\mathrm{Nb} \\
\text { of spect. }\end{array}$ & $<A_{V}>$ & $A_{V} \min$ & $A_{V} \max$ & Disp \\
\hline FSM & 33 & 2.88 & 0.18 & 7.1 & 1.5 \\
IRAS & 17 & 2.71 & 0.51 & 6.8 & 2 \\
\hline
\end{tabular}
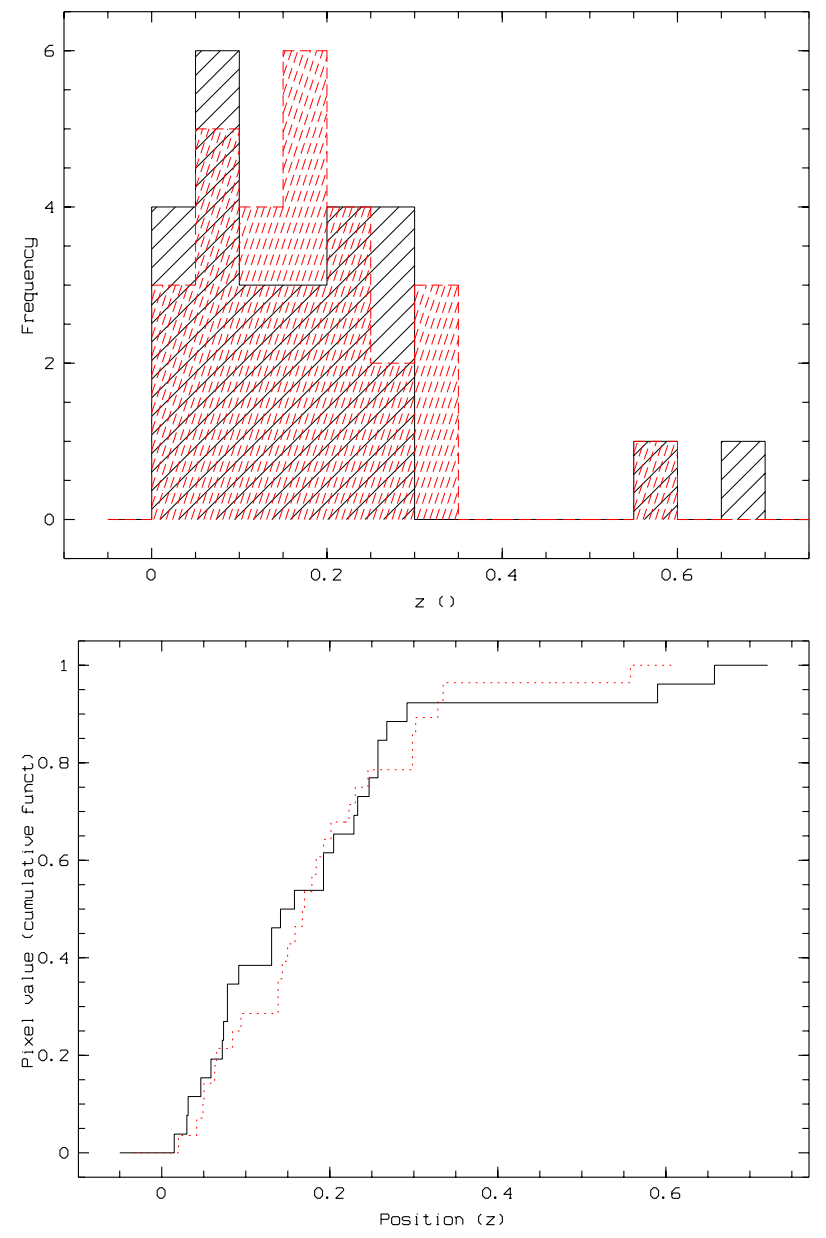

Fig. 4. Distribution of the redshifts of the IR sources.

of $50(\tau([\mathrm{OII}])=4)$ for the [OII] blend, as compared to a factor of 2 in $\mathrm{H} \alpha$ and 4 in [OII] when we take the usual prescription of $A_{V}=1$ only. It is thus important to characterize the type of object selected, in order to apply the appropriate correction.

\subsection{Redshift distribution}

We show in Fig. 4 and Table 5 the statistics of the obtained redshift distributions. It is quite obvious that the repartitions are similar. The Kolmogorov-Smirnov test tells us that two stochastic samples taken from the same parent distribution have a probability of more than $70 \%$ to be more different than these two.

The only two objects at larger redshift $(z>0.3)$ in the FSM sample are Seyfert galaxies. The brighter sources in the $170 \mu \mathrm{m}$ selection are thus rather close by.
Table 5. Statistics on $z$ for both samples. The two distributions are obviously very alike.

\begin{tabular}{llllll}
\hline \hline $\begin{array}{l}\text { Sample } \\
\text { name }\end{array}$ & $\begin{array}{l}\mathrm{Nb} \\
\text { of spect. }\end{array}$ & & & & \\
\hline FSM & 26 & 0.18 & 0.015 & 0.658 & 0.16 \\
IRAS & 28 & 0.18 & 0.020 & 0.558 & 0.12 \\
\hline
\end{tabular}

\subsection{Emission lines and optical properties}

We will use classical diagnostic diagrams to derive the nature of those galaxies.

In the FSM sample, we usually have secure measurement for (at least) the following lines: [OII] (3727 $\AA$ ), $\mathrm{H} \beta$, [OIII] (4959 ̊, $5007 \AA), \mathrm{H} \alpha$, [NII] (6548 $\AA, 6583 \AA)$. There is no broad emission line object in the FSM sample. Although our statistics is small (only 20 objects in the brighter part studied here), this is consistent with the results of the BGS where Veilleux et al. (1995) found only two Seyfert 1 galaxies out of the 140 spectra analyzed, and they are very bright IR objects.

The fraction of emission-line galaxies in our sample is very high, confirming the pertinence of an IR-selected sample to find star forming objects. To all but one (FSM_18) $170 \mu \mathrm{m}$ sources, the optical corresponding object is one (or several) emission-line galaxy. Except for this single case, all spectra can be classified "emission + absorption" (all spectra have Balmer emission, most have Balmer absorption and metallic absorption lines), see Fig. 9.

Figure 5 presents one of the most classical diagnostic diagrams (Baldwin et al. 1981) to separate HII region-type galaxies (bottom left of the diagram) from Seyfert 2 (upper right of the diagram) and LINER (Low Ionisation Nuclear Emission Line Regions, bottom right). The frontiers are from Dessauges-Zavadsky et al. (2000), but a variety of empirical separations can be found in the literature, and the definition of the regions is rather approximative.

This diagram is well adapted to our data. The signal to noise ratio of our spectra does not allow us to use [OI] or [NeIII] lines (usually not detected), or the [SII] often affected by atmospheric features. For objects at $z>0.3, \mathrm{H} \alpha$ is out of the observed range, so we use also the "R23" criterion from Dessauges-Zavadsky et al. (2000):

$R 23=\frac{[\mathrm{OII}]_{3727}+[\mathrm{OIII}]_{4959}+[\mathrm{OIII}]_{5007}}{\mathrm{H} \beta}$

and $R 23>1.1$ for Seyfert 2 objects.

One object is classified "Seyfert 2" by both criteria: FSM_004. The "R23" test points out to two more objects, FSM_011 and FSM_035, for which $z>0.5$ and thus we don't have the $\mathrm{H} \alpha$ line. These latter two objects are quasi-stellar (punctual appearance on acquisition image) and at intermediate redshift $(z \simeq 0.6)$; they are the most remote objects of our sample. The excitation of FSM_11 (and absence of broad $\mathrm{H} \beta$ ) indicates a Seyfert 2 galaxy, while FSM_35/1 is clearly a QSO. FSM_4 is a nearby active galaxy at $z=0.0784$.

Two FSM objects are classified as "LINER" on the diagram, FSM_013 and FSM_003 (though this last one is not secure, given the error bars). Unfortunately, the classical test for 


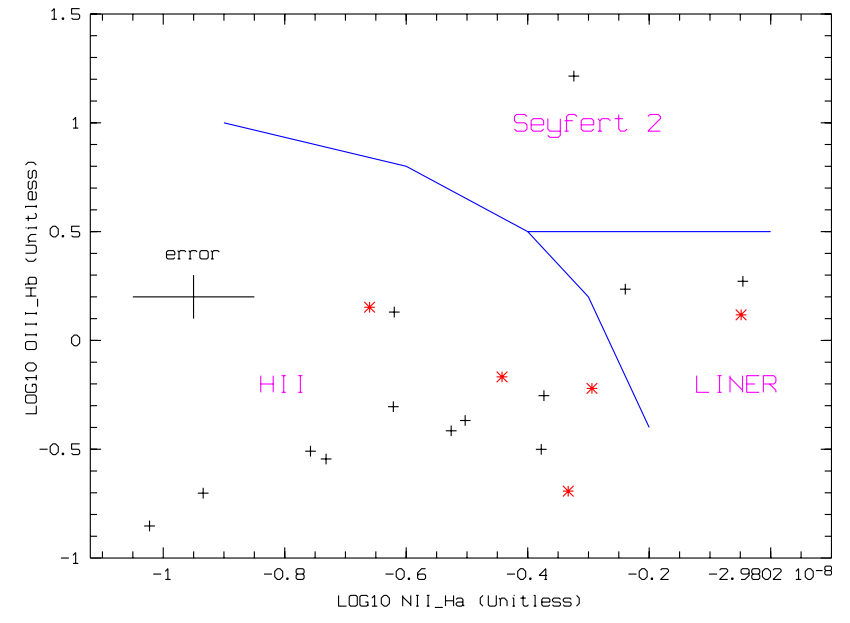

Fig. 5. Diagnostic diagram for both our samples. +, FSM sample; *, IRAS reference sample. All emission-line measurements are corrected for dust extinction and absorption lines. Only spectra with $\mathrm{H} \beta$, [OIII], $\mathrm{H} \alpha$ and $[\mathrm{NII}]$ can be plotted: due to a smaller spectroscopic range, very few IRAS objects could be plotted (see text). The cross at the left indicate the average error bar.

LINER is not applicable here since the [OI] $6300 \AA$ line has not been detected in our spectra.

We thus get 3 to 5 active objects out of 29 in the FSM sample, that is $11 \%$ to $19 \%$ of the objects. The IRAS reference sample shows similar results, though the statistic is much smaller due to the smaller observed range and spectral resolution.

These values are similar to the CFRS results (between 8\% and $18 \%$ when including the effects of stellar absorption), but rather far from the high number of AGN found in the IRAS bright galaxy sample. If this effect is confirmed (by larger samples) it tends to show that the faint IR galaxies are indeed a population different from the bright IR objects: heavily obscured, but with a majority of star forming objects and only few AGN. This is consistent with the finding (e.g. Lutz et al. 1998; Veilleux et al. 1999) that the fraction of AGN increases strongly when going to ULIRG objects. Indeed, as shown in Sect. 5.5, the IR luminosities of the bright FSM objects are rather modest $\left(L_{\mathrm{IR}}<10^{12} L_{\odot}\right)$ and, at those luminosities, the fraction of AGN's is smaller than $20 \%$ in the 1 Jy sample studied by Veilleux et al., for instance. It increases abruptly to $50 \%$ only at $L_{\mathrm{IR}}>10^{12.3} L_{\odot}$.

\subsection{IR temperatures}

One of the main characteristic of our galaxies is their cool IR temperature, which can be determined when we have more than one observational point. As indicated in Table 1, for 5 out of 29 objects, we have, in addition to the $170 \mu \mathrm{m}$ fluxes, also the 60 and $100 \mu \mathrm{m}$ IRAS measurements, obtained from the SCANPI procedure. For another 13 galaxies, this method gives only upper limits for 60 and/or $100 \mu$ m fluxes.

To characterise the dust emissivity of these galaxies, we studied three quantities: the greybody temperature $T$, the infrared colors and the spectral energy distribution.

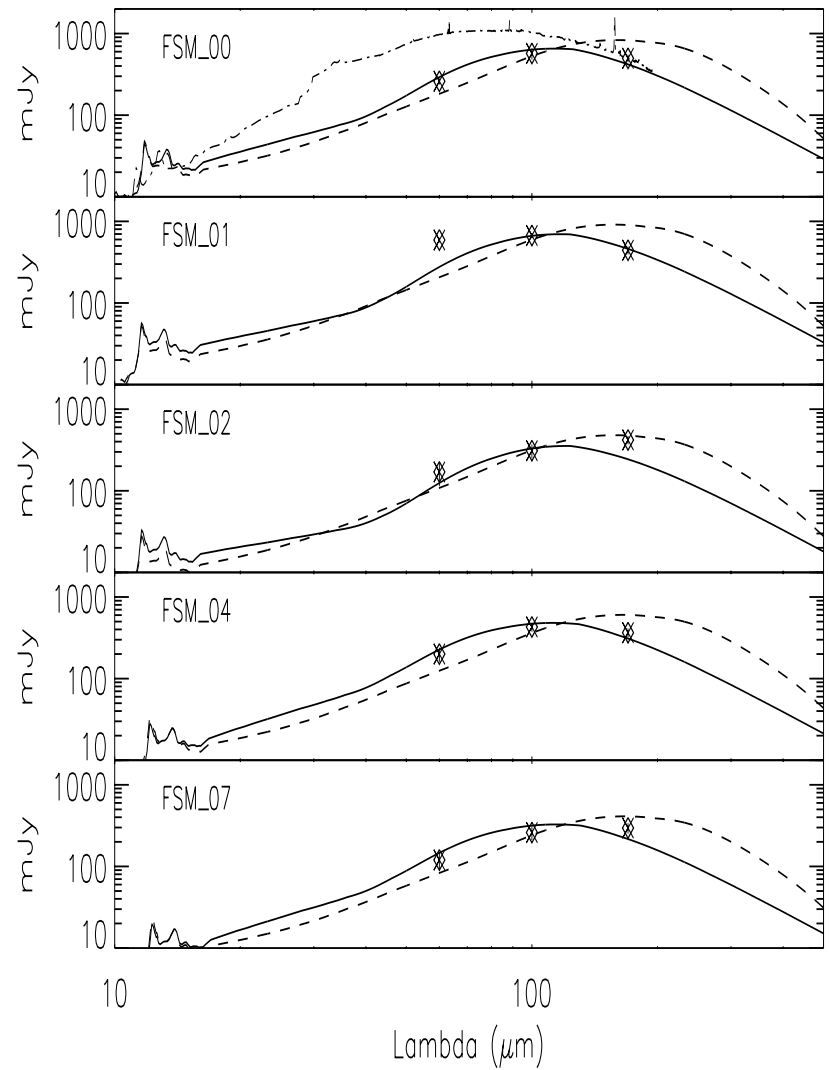

Fig. 6. SED modelisation compared to five observed cases. Solid line: starburst template, dashed line: Cold template. Both templates are from Lagache et al. (2003). Also shown for comparison is the spectrum of M82 (dashed-dotted).

The greybody temperature $T$ was computed for direct comparison with other galaxies from the literature. We use a spectral index of $\beta=1.5$, and fit a modified blackbody to the infrared measurements at 60 and $170 \mu \mathrm{m}$ for 5 galaxies, and use the upper limits at $60 \mu \mathrm{m}$ for 13 other galaxies. We find an average temperature of $22.7 \mathrm{~K}$, with values ranging from $20.9 \mathrm{~K}$ (FSM_002) to 24.7 (FSM_001) for the first 5 galaxies, and a somewhat wider distribution when we work also with upper limits: average temperature of $23.5 \mathrm{~K}$ with values ranging from 19.5 (FSM_005) to 32.7 K (FSM_011). This results indicate a rather cold population.

In addition to the greybody temperature, we computed the IR color $\log \left(S_{v}(100 \mu \mathrm{m}) / S_{v}(60 \mu \mathrm{m})\right)$, as usually done for IRAS galaxies. We find a mean ratio of -0.32 , with a dispersion of 0.2 dex. This is highly compatible with the "normal" IRAS galaxies: Soifer et al. (1989; their Fig. 1) show that the histogram of the color distribution of all the BGS galaxies is centered at -0.3 . A similar value is obtained with our own small IRAS sample.

Thus the FSM galaxies have the same average color as normal IRAS $60 \mu \mathrm{m}$ selected galaxies, but the observations at $170 \mu \mathrm{m}$ show a cooler dust temperature. This is generally the result whenever a longer wavelength point is added to IRAS measurements, as a single temperature blackbody is probably too crude an approximation to reproduce the SED of those objects. 
To investigate further the IR spectral energy distribution of these galaxies, we fit our observational data points with the "starburst" and "normal" galaxy templates from Lagache et al. (2003). The "normal" galaxy template is colder than the "starburst" template, with a peak at around $180 \mu \mathrm{m}$. Results of the fit are shown in Fig. 6 and detailed below:

- FSM_000: the best fit is obtained with the "starburst" template with $L_{\mathrm{FIR}}=10.95$;

- FSM_001: the best fit is obtained with the "starburst" template with $L_{\text {FIR }}=10.6$. The $60 \mu \mathrm{m}$ point is however not well reproduced: this can be due to the existence of a hotter component;

- FSM_002: the best fit is obtained with the "normal" template with $L_{\mathrm{FIR}}=10.35$;

- FSM_004: the best fit is obtained with the "starburst" template with $L_{\mathrm{FIR}}=11.25$;

- FSM_007: Both templates give only an approximate reproduction of the measured fluxes.

Note that none of these galaxies has a SED which resembles the one of M 82. Although the sample here is small, it appears that our objects are best reproduced by a cold starburst template, giving luminosities typical of LIRGs.

\subsection{IR luminosity}

As for most objects in our sample, we do not have the IRAS $(60 \mu \mathrm{m}, 100 \mu \mathrm{m})$ flux measurements, we cannot use the classical formula (Helou et al. 1988) to derive the total IR luminosity $\left(L_{\mathrm{IR}}\right)$. We are thus obliged to use models or template SED to derive it in a homogeneous way for all objects in the sample.

We first performed a series of tests to determine the best way to compute the total luminosity. Three different methods are compared (we note the disadvantages of each method in italics):

1 - take in the grid of spectra proposed by Lagache et al. the one that is closest to the observed points. This supposes a universal relationship between luminosity and color.

2 - use the normal template SED from Lagache (built from a number of FIRBACK objects). This supposes a universal shape for all objects.

3 - fit only the shape of the model of Lagache et al., regardless of its intrinsic absolute value at peak, and then compute the total luminosity. This method is correct only for those objects were we have more than one observational point ( 9 for the FSM sample, 3 for the IRAS reference sample).

Though the three methods lead to very different colors for a given object, the computed total luminosities do not differ by more than $20 \%$ (and $10 \%$ on average), which is also the value of the error bars of the ISO and IRAS data. We conclude that any of the two first methods can be used to derive the total luminosity for all objects in a homogeneous way, with a resulting uncertainty of less than $20 \%$. The third method can be used as a check of this uncertainty for the 6 objects where more than one measured point is available. Note that this "total" IR luminosity is about 2 times larger than the FIR usually derived from the IRAS data alone (40-200 $\mu \mathrm{m}$; Helou et al. 1988).

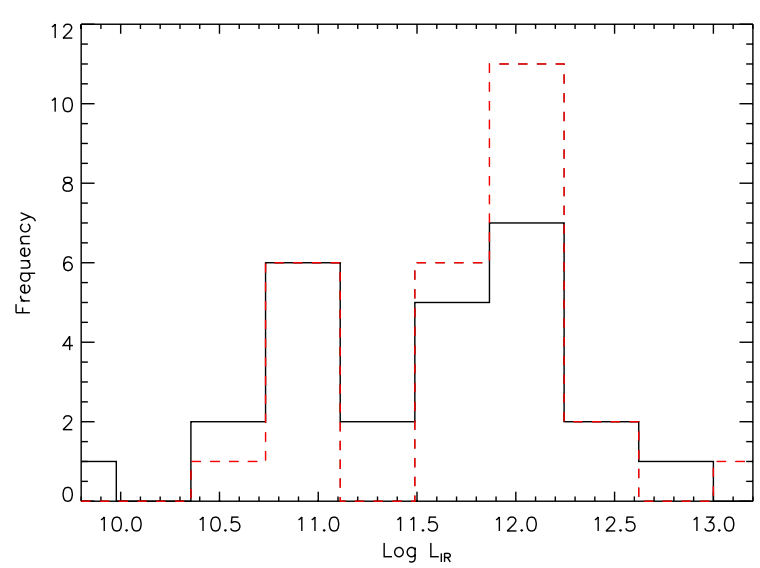

Fig. 7. Distribution of IR luminosities (from 0.4 to $1000 \mu \mathrm{m}$ ) for i) the FSM sample (solid histogram) and ii) the IRAS sample (dashed histogram).

Table 6. Statistics on total IR luminosities (from 0.4 to $1000 \mu \mathrm{m}$, in units of solar luminosity) for both samples.

\begin{tabular}{cccccc}
\hline \hline $\begin{array}{c}\text { Sample } \\
\text { name }\end{array}$ & $\begin{array}{c}\mathrm{Nb} \\
\text { of spect. }\end{array}$ & $\begin{array}{c}\text { average } \\
\log \left(L_{\mathrm{IR}}\right)\end{array}$ & $\begin{array}{c}\log \left(L_{\mathrm{IR}}\right) \\
\min \end{array}$ & $\begin{array}{c}\log \left(L_{\mathrm{IR}}\right) \\
\max \end{array}$ & $\begin{array}{c}\sigma \\
(\mathrm{dex})\end{array}$ \\
\hline FSM & 26 & 11.5 & 9.6 & 12.9 & 0.8 \\
IRAS & 27 & 11.7 & 10.6 & 13.0 & 0.6 \\
\hline
\end{tabular}

$L_{\mathrm{IR}}$ has in the end been determined using the first method, and the error is then of the order of 0.1 dex.

Most of the FIR selected galaxies are LIRGs (Luminous Infrared Galaxies) with $10^{11}<L_{\mathrm{IR}}<10^{12} L_{\odot}$, and many of them have lower luminosities, down to $L_{\mathrm{IR}} \simeq 10^{10.5}$. Very few are ULIRGs: the only two objects belonging to this category are the higher redshift AGN's found here in the FSM. Figure 7 shows the distribution of $L_{\mathrm{IR}}$ and Table 6 gives the statistics on $L_{\mathrm{IR}}$.

We finally derived the IR to optical luminosity ratio as follows:

- $L_{\mathrm{IR}}$ computed with the first method;

- optical luminosity computed by integrating the continuum from 4000 to $7000 \AA$ in the rest frame (range directly accessible with our spectra). We find ratios between 10 and 100, with an average of 65 . These are rather high values, indicative of high extinction and/or strong activity. On the two higher redshift FIRBACK source identifications $(z=0.45$ and $z=0.91)$ found in FN1, Chapman et al. (2002) derived these ratios between 10 and 15; but correcting for the different definition they used (standard IRAS FIR, and a 4 times broader range for the optical luminosity), it appears that their values are entirely compatible with those we find here in this larger sample.

\subsection{Star formation}

Several indicators are generally used to determine the star formation rate of galaxies, the two most useful ones in our sample being the $\mathrm{H} \alpha$ emission line and the IR luminosity.

The $\mathrm{H} \alpha$ emission line fluxes used to derive the star formation rate are corrected for extinction: this is indispensable as 
Table 7. Distribution of the star formation rate for the FSM and the IRAS sample (computed from the corrected $\mathrm{H} \alpha$ emission flux and from the total IR luminosity; only objects classified as "HII regionlike" in Sect. 4.3 have been used). For comparison, the SFR inferred from the IR is also shown for the sub-samples with a measurable $\mathrm{H} \alpha$ line.

\begin{tabular}{|c|c|c|c|c|}
\hline \multicolumn{5}{|c|}{ Star formation rates $\left(M_{\odot} / \mathrm{yr}\right)$ from corrected $\mathrm{H} \alpha$ flux } \\
\hline & sample size & average & $\min$ & $\max$ \\
\hline FSM & 20 & 9.7 & 0.3 & 39 \\
\hline IRAS & 20 & 37 & 0.5 & 440 \\
\hline \multicolumn{5}{|c|}{ Star formation rate $\left(M_{\odot} /\right.$ yr) from IR luminosity } \\
\hline \multirow{3}{*}{$\begin{array}{l}\text { FSM } \\
\text { (with } \mathrm{H} \alpha \text { ) }\end{array}$} & sample size & average & $\min$ & $\max$ \\
\hline & 21 & 41 & 0.5 & 990 \\
\hline & 20 & 41 & 0.5 & 320 \\
\hline IRAS & 27 & 65 & 2.6 & 1300 \\
\hline (with $\mathrm{H} \alpha$ ) & 20 & 106 & 5.2 & 340 \\
\hline
\end{tabular}

our objects are heavily obscured. For the conversion factor, we use the values from Kennicutt (1983):

$\operatorname{SFR}\left(M_{\odot}\right.$ per year $)=\frac{L_{\mathrm{H} \alpha}\left(10^{41} \mathrm{erg} / \mathrm{s}\right)}{1.12}$.

To compute the star formation rate from the IR luminosity, we use the calibration of Devriendt et al. (1999) which is consistent with the SED models used:

$\operatorname{SFR}\left(M_{\odot}\right.$ per year $)=\frac{L_{\mathrm{IR}}\left(10^{9} L_{\odot}\right)}{7.7}$.

The results for both samples are presented in Table 7. The correction applied to the $\mathrm{H} \alpha$ flux seems to be insufficient, as the $S F R$ is lower than the one calculated from the IR luminosity: the star forming regions are probably completely screened in the optical, as suggested by the rather high values of optical depths derived for $\mathrm{H} \alpha$ in Sect. 4.1. However, the objects we observed do not have large Star Formation Rates: with the exception of one or two more "extreme" objects, the average $S F R$ is a few tens of solar masses per year, which corresponds to moderate starburst episodes.

\section{Discussion on the nature of the galaxies}

We observed the brightest objects in the FIRBACK South Marano sample, and were almost complete in our follow-up of the brightest sources (20 out of 22 IR sources in the $4 \sigma$ catalog). Pending the follow-up of the faintest objects in the FSM complementary catalog ( 15 objects with $3<\sigma<4$ ), and the study of similar sources in the northern FIRBACK fields, we have already here with this sample a fairly good picture of the nature of the brightest $170 \mu \mathrm{m}$ sources.

Because of the so-called "negative K-correction effect" (e.g. Dole et al. 2001), a deep flux limited survey in a far-IR band like FIRBACK is expected to be biased towards distant galaxies. Our results however point to a population of galaxies much nearer than expected. Sajina et al. (2003) described the $T /(1+z)$ degeneracy in imaging infrared surveys such as FIRBACK. The results of our follow-up shows that the $170 \mu \mathrm{m}$ luminosity is more an effect of low temperature than one of high redshift, at least for the brightest objects. This has also been seen by Chapman et al. (2002), who, upon selecting just two FIRBACK objects supposed to be at very high redshift, found in fact redshifts much lower than expected. We show that this property is more general and that we systematically overestimated the sources's redshifts.

The physical properties of the galaxies in this sample can be summarized as follows.

First, as expected, they are heavily extincted. Measurements of $A_{V}$ give an average value of about 3, with extreme cases up to 7 , consistent with classical IRAS galaxies (Sanders \& Mirabel 1996). A very high proportion (more than $80 \%$ ) of the galaxies shows narrow emission lines. The origin of the IR emission at $170 \mu \mathrm{m}$ is therefore likely to be a central/circumnuclear engine heating the dust, just as in IRAS galaxies. The total infrared luminosity produced by this source is moderate in both our IRAS faint galaxies and in the FIRBACK galaxies: they can be classified as "luminous infrared galaxies", with $L_{\mathrm{IR}} \simeq 10^{11} L_{\odot}$, but not ULIRG's. The dominant energy source is star formation in most cases ( $\simeq 85 \%$ of the galaxies), as indicated by the analysis of the emission spectrum. Among the $15 \%$ of objects possibly dominated by an AGN, two are the most luminous and farthest objects of the sample; only one is dominated by an active nucleus in the $4 \sigma$ sample consisting of nearby, bright objects. Consistently with the properties of IRAS galaxies, we find that: i) nearby luminous infrared galaxies are mainly powered by star formation and ii) the proportion of AGN increases with the IR luminosity.

The homogeneity of the properties of $80 \%$ of the FIRBACK galaxies analyzed so far allows us to consider them as a population in itself, characterized by the following properties: a small redshift $(z \simeq 0.1)$, a moderate IR luminosity $\left(L \simeq 10^{11} L_{\odot}\right)$, a peak of infrared emission between 100 and $200 \mu \mathrm{m}$, and a main energy source due to star formation with moderate SF rates $\left(S F R \simeq 10 M_{\odot} / \mathrm{yr}\right)$. Is this population "new" or is it part of another, already known, class of galaxies? To answer this question, we can compare this FSM sample to three other samples of infrared galaxies: the $15 \mu \mathrm{m}$-selected sample of Elbaz et al. (2002), our sample of faint IRAS galaxies, and the classical IRAS galaxies in the BGS (Soifer et al. 1987; Kim et al. 1995; Veilleux et al. 1995, under others).

The comparison with the galaxies of Elbaz et al. (2002) in the ISOCAM $15 \mu \mathrm{m}$ survey shows that the two population differ rather widely. Elbaz et al. found a higher density of objects in the HDF-N (40 galaxies at $S_{15}>0.1 \mathrm{mJy}$ over a field of $26^{2}$ ), but their survey is also deeper: $0.1 \mathrm{mJy}$ at $\lambda=15 \mu \mathrm{m}$ corresponds to $4.5 \mathrm{mJy}$ at $\lambda=170 \mu \mathrm{m}$ with our template SED, and this leads to galaxies more luminous on the average, and at higher redshift $(<z>=0.8)$. But the FSM galaxies cannot be simply interpreted as corresponding to the closest and coldest tail of the distribution of Elbaz's objects: those have a very low infrared luminosity and usually no emission line, contrary to the FSM galaxies. The FSM galaxies resemble in fact much more to their highest redshift objects. The two populations are therefore quite different. The comparison can however not be pushed much further, in particular the comparison of the SED's, as no far-IR measurements are available for Elbaz's sample. 

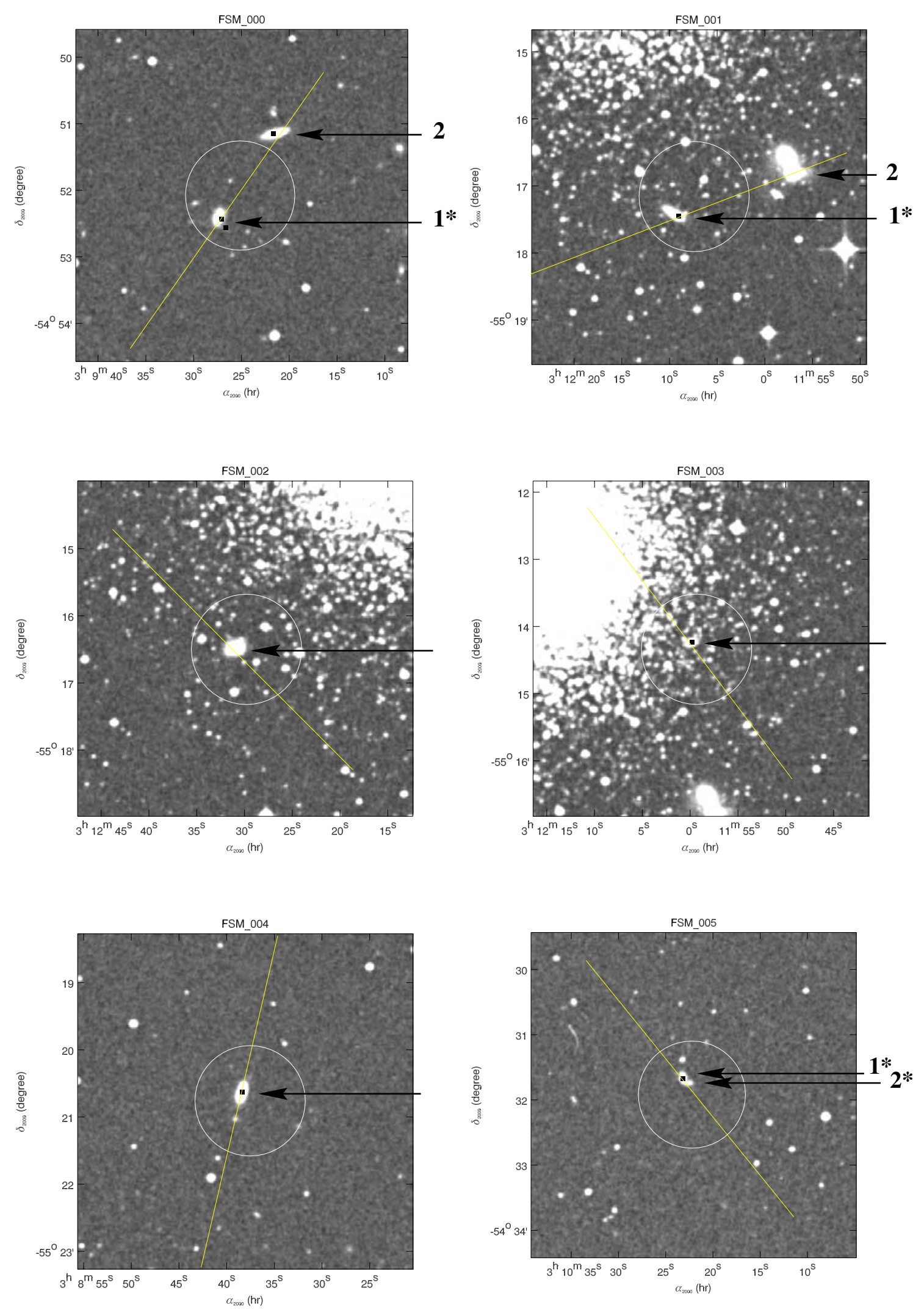

Fig. 8. Identification charts for FIRBACK South Marano field. DSS optical image superimposed to the error circle of infrared ISO source and radio emission (black mark). The straight line shows the position of the slit (1.2" large and 5' long), and an arrow indicates a galaxy with an available spectrum. The number associated to each arrow corresponds to the number in Table 2, with an asterisk indicating that the galaxy is associated to the infrared emission. 

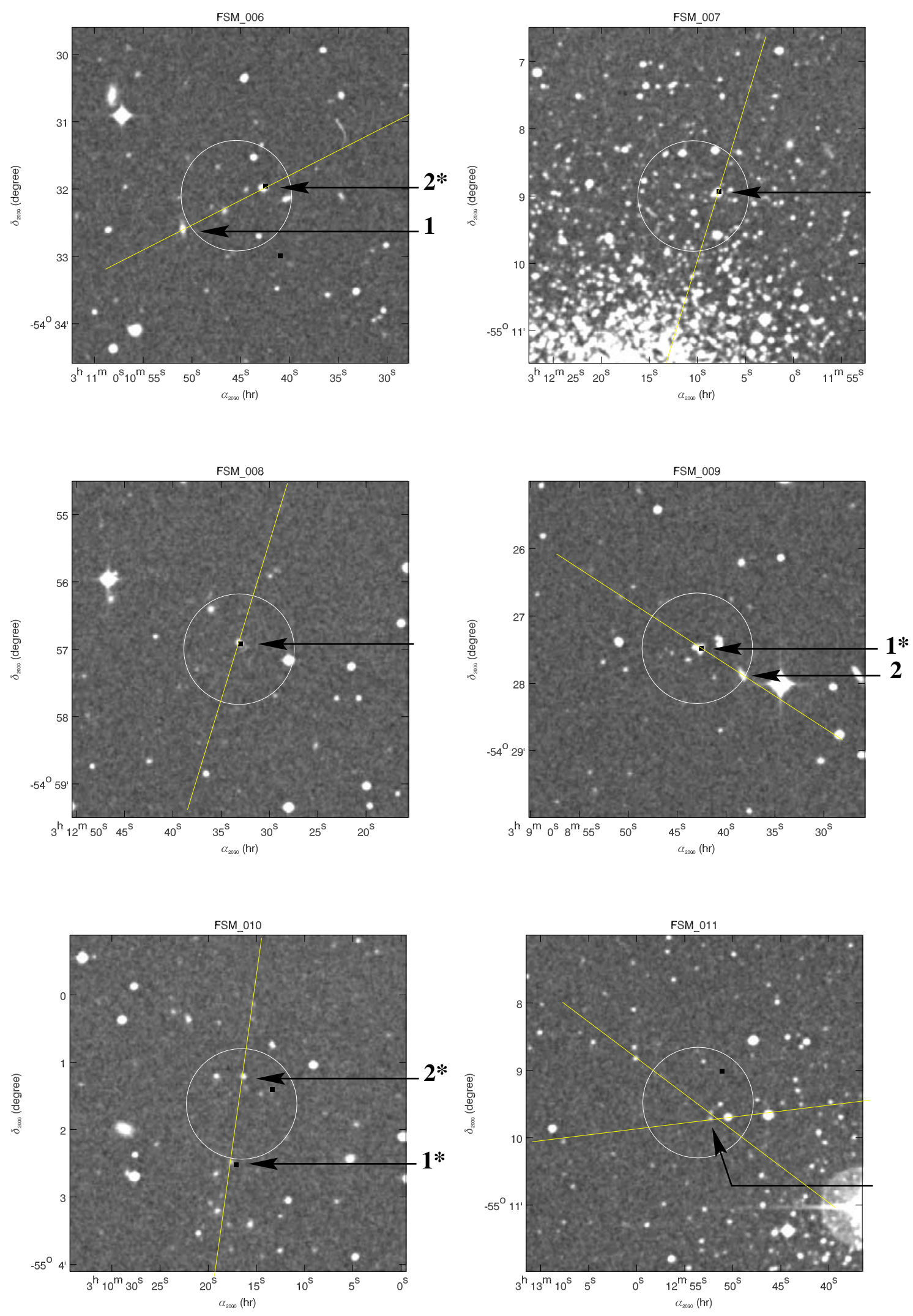

Fig. 8. continued. 

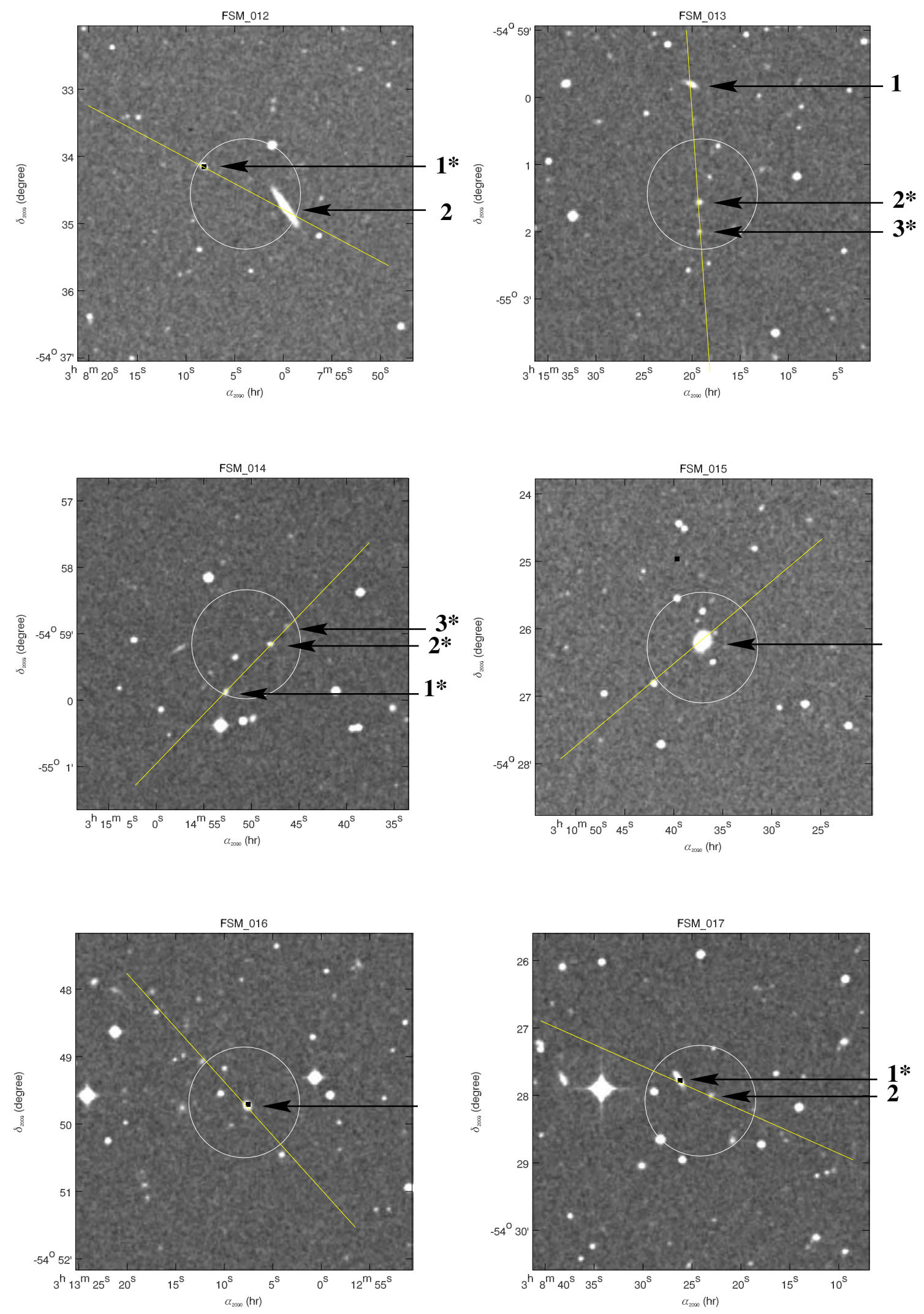

Fig. 8. continued. 

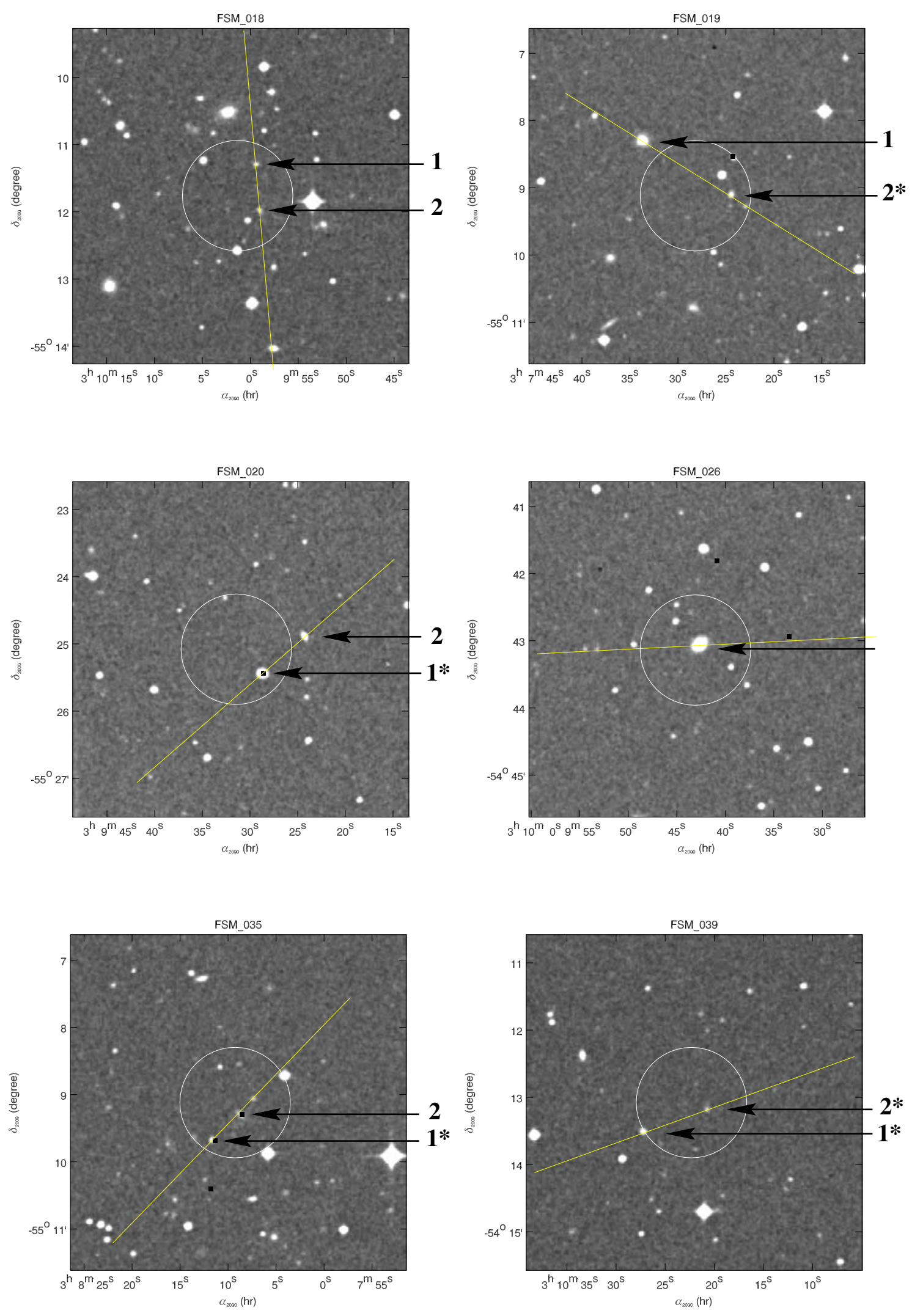

Fig. 8. continued. 

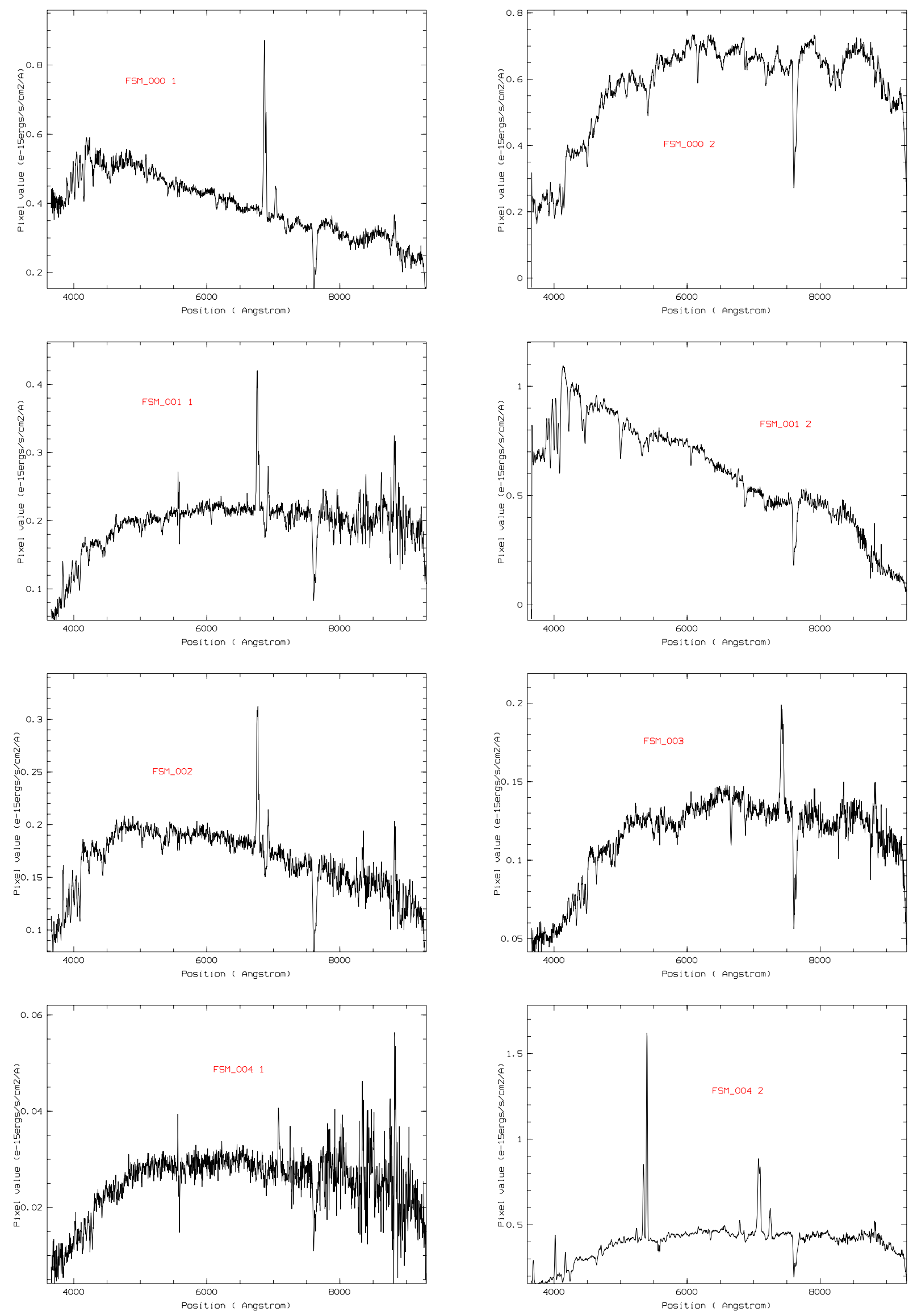

Fig. 9. Spectra. 

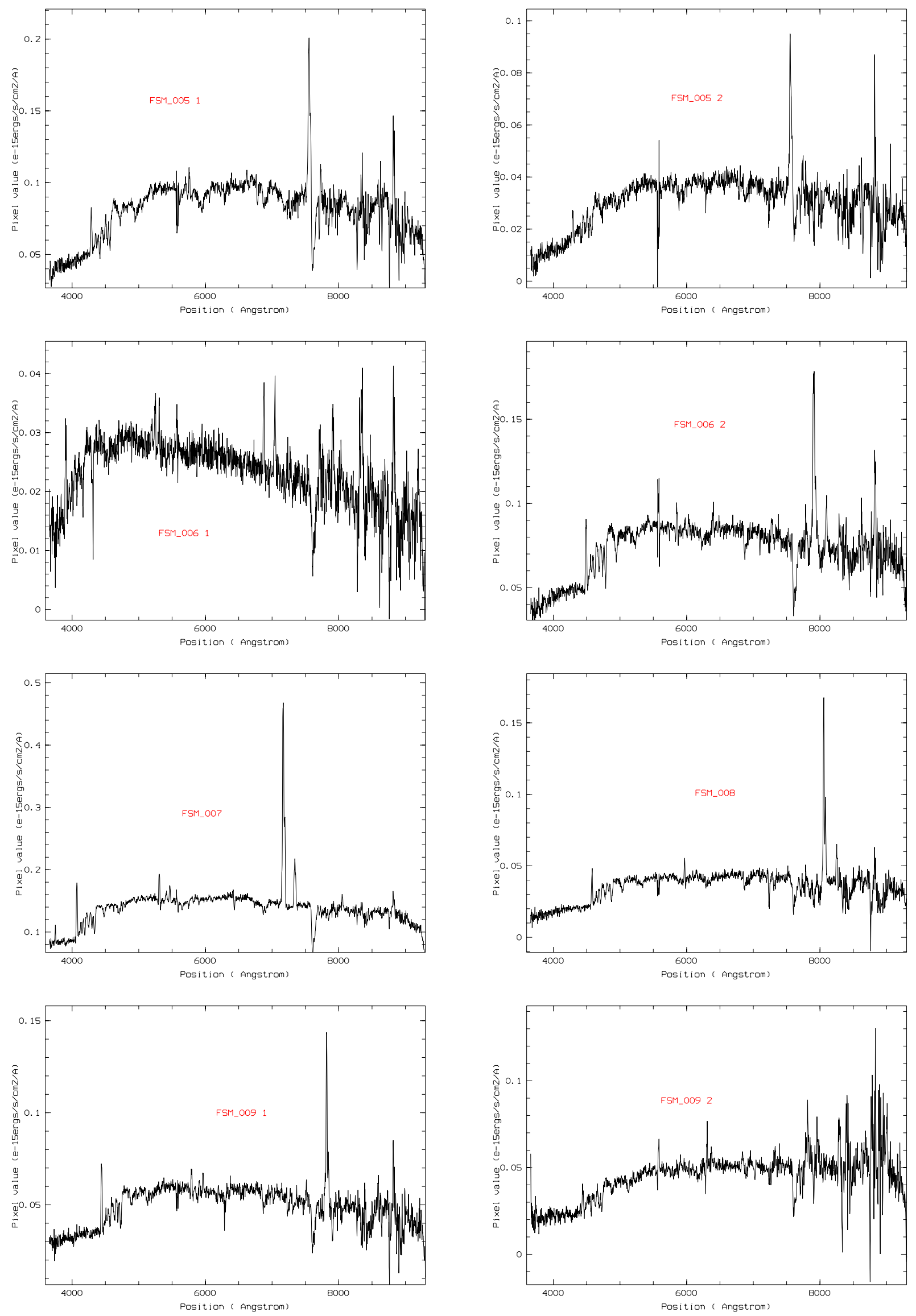

Fig. 9. continued. 

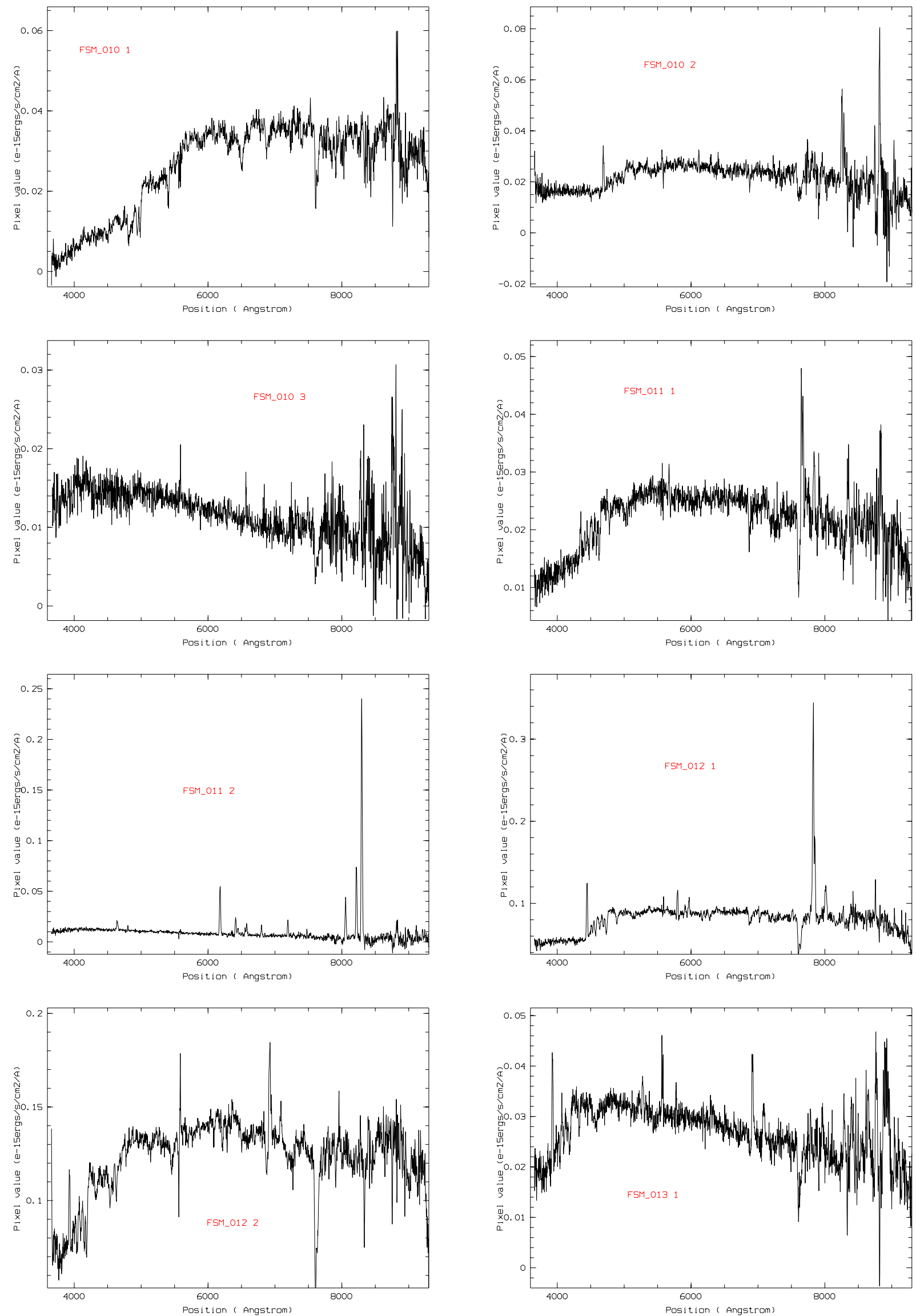

Fig. 9. continued. 

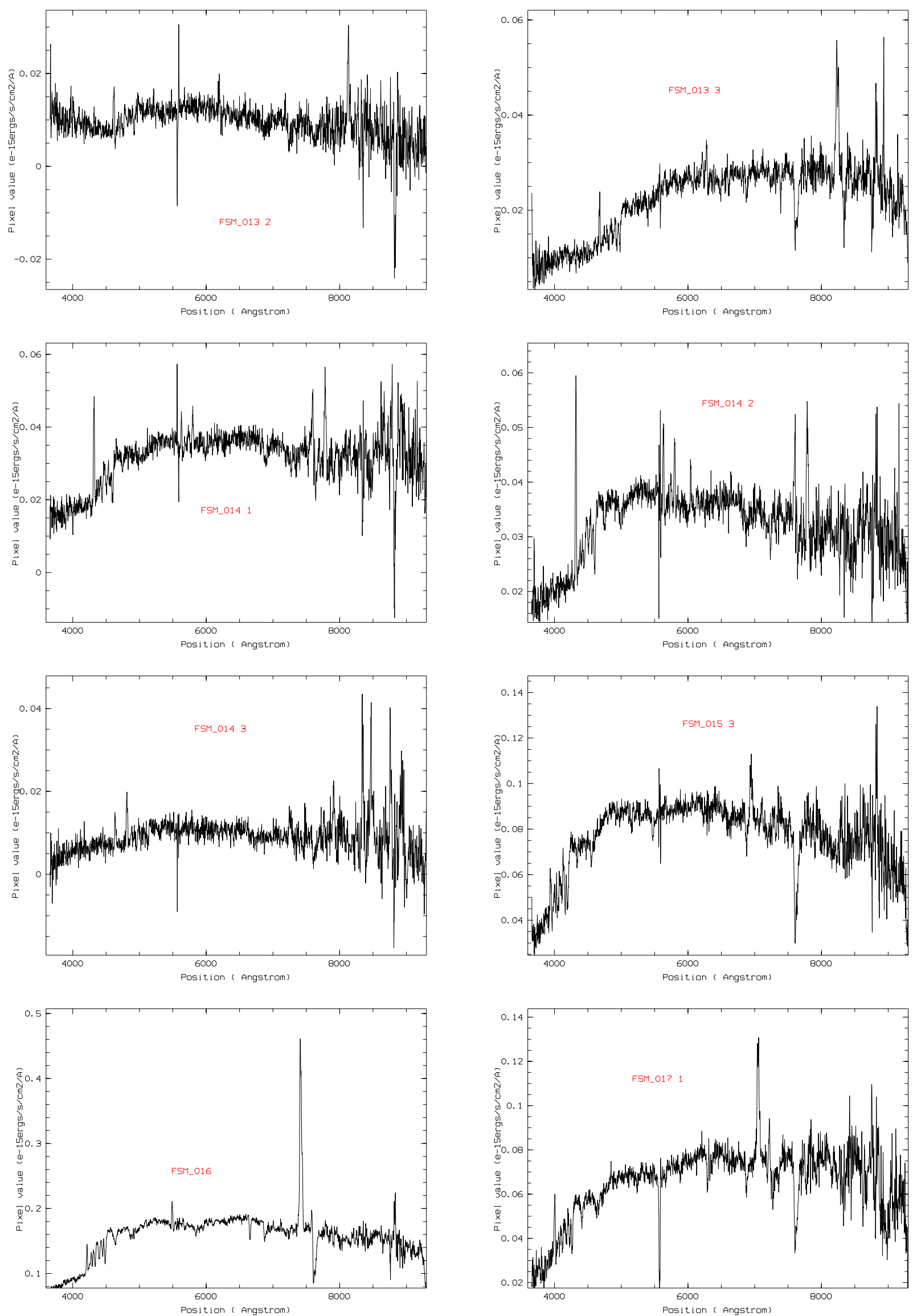

Fig. 9. continued. 

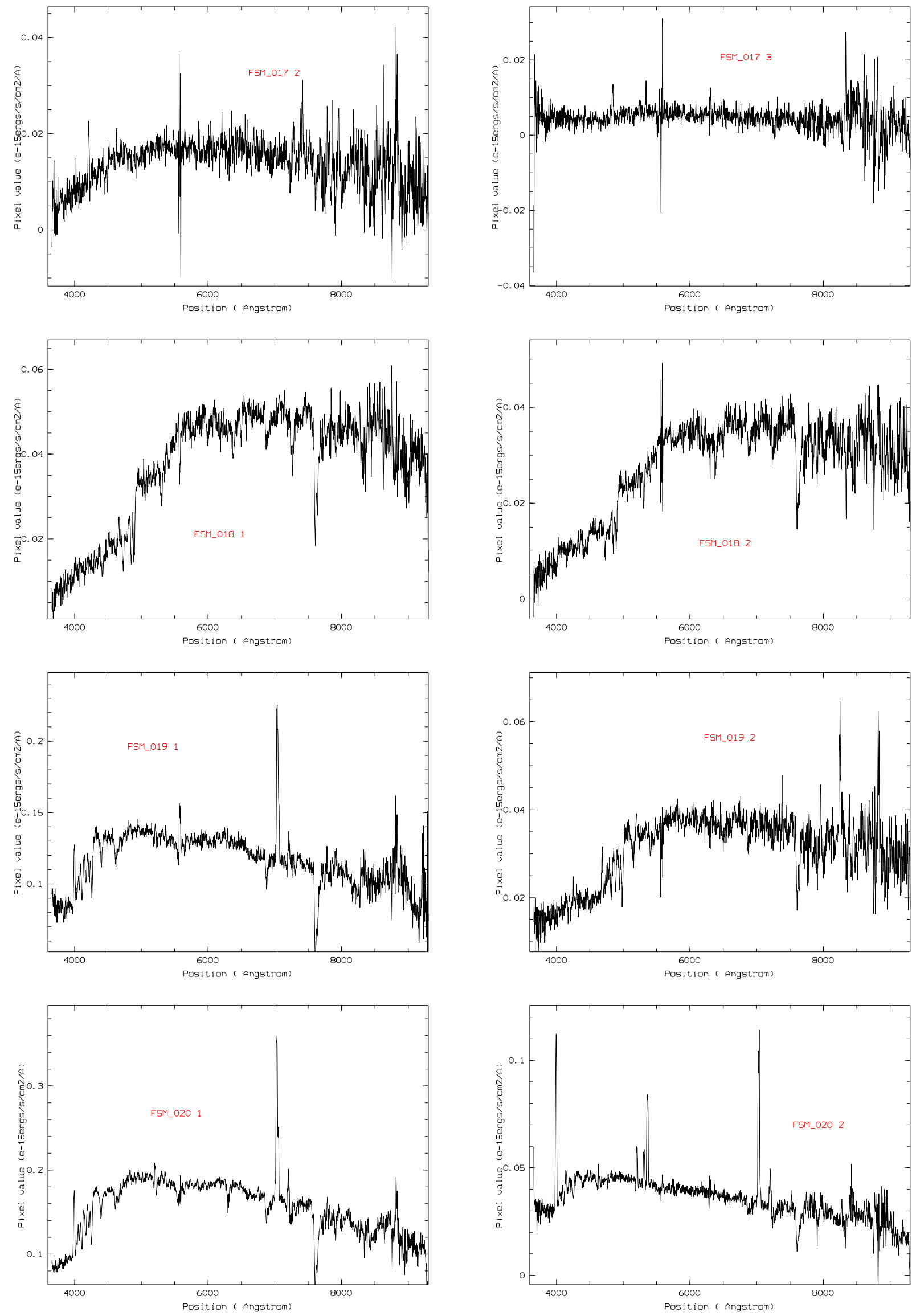

Fig. 9. continued. 

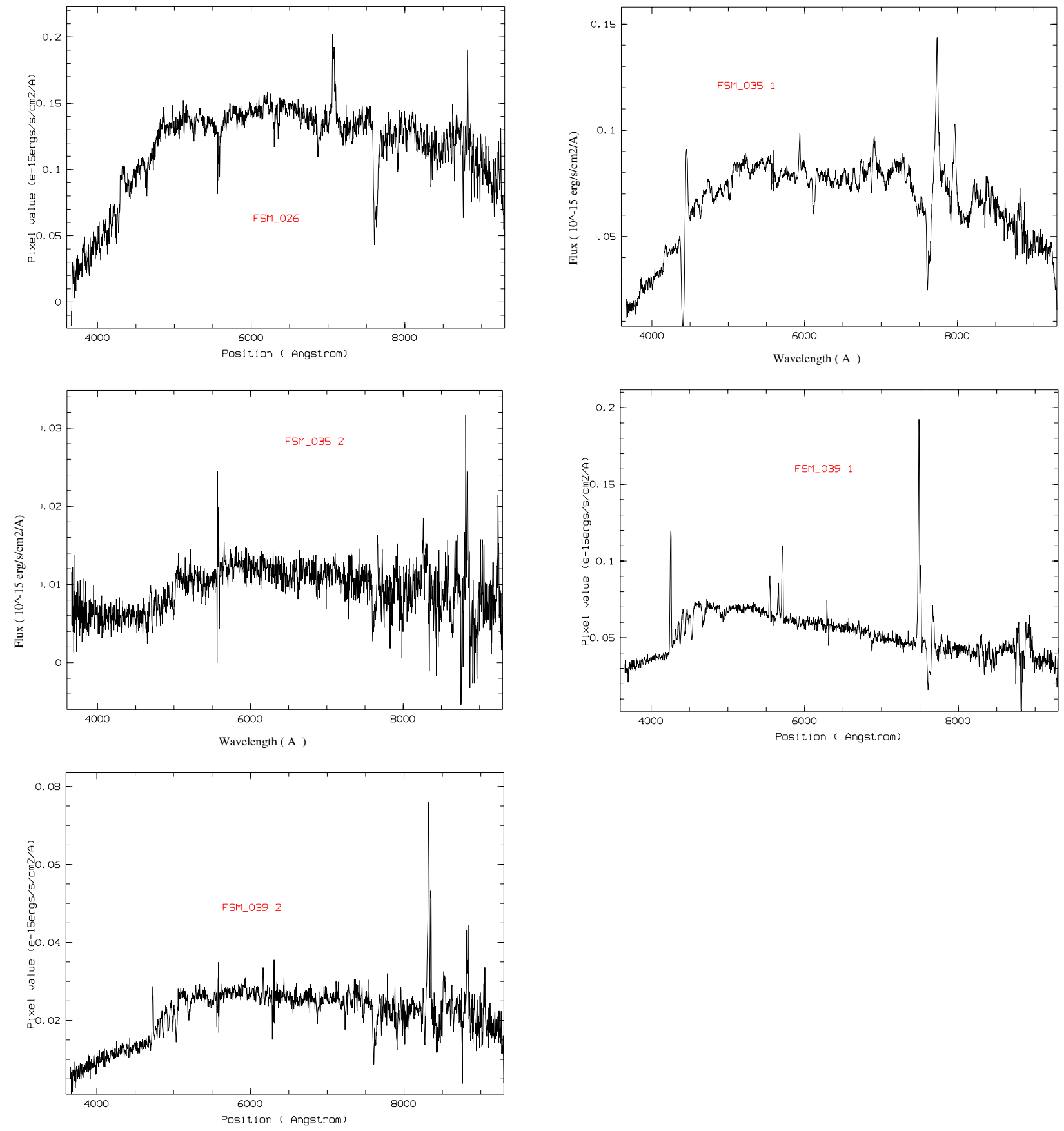

Fig. 9. continued.

On the contrary, the comparison of the FSM galaxies with the very faint IRAS galaxies selected at $60 \mu \mathrm{m}$ shows that the two populations are very similar. All the properties we were able to measure on both samples are close to identical: $z$ distribution, average IR luminosity, nature of the powering source, and star formation rates. With the available data, it is impossible to distinguish statistically between the two samples. Unfortunately, because this IRAS sample corresponds to the faintest detectable galaxies (Bertin et al. 1997) at $60 \mu \mathrm{m}$, which is the most sensitive band in the IRAS survey, no $100 \mu \mathrm{m}$ data are available for them and therefore no dust temperature estimate can be made, nor a SED derived. Further measurements with SIRTF will be desirable to better characterize those objects.

If we now compare the FSM galaxies with the more classical IRAS galaxies like the BGS for example, we find many similarities (spectral properties, IR luminosities) but one significant difference: the shape of the infrared SED. Other deep samples of IRAS galaxies have focused on the search for ULIRG's and therefore selected, by definition, objects much warmer than the FSM galaxies. It is therefore quite plausible that the majority of faint, unstudied, IRAS galaxies have on average a colder IR emission than assumed up to now (and than measured in the few $60 \mu \mathrm{m}$ selected samples). Although we 
cannot exclude at this stage of analysis that the galaxies picked up here at $170 \mu \mathrm{m}$ and the very faint IRAS galaxies have in fact an even colder infrared emission than the main IRAS sample, it has been shown in the ISOPHOT serendipity sample by Stickel et al. (2000) that a cold dust component was present in many galaxies, so that all these objects could be similar, but only a detailed analysis of SED's will definitely answer this question.

\section{Conclusion}

The brightest objects of the FIRBACK South Marano survey sample the same population as the IRAS faint survey: a numerous population of cold, dusty, nearby galaxies. These galaxies are characterized by:

i) a high density of sources (about 20 per square degree) in a small volume of the universe, since all these sources lie at $z \simeq 0.2$;

ii) a cold IR emission, with a peak of the emission closer to $170 \mu \mathrm{m}$ than to $100 \mu \mathrm{m}$;

iii) a moderate IR luminosity in the LIRG range $\left(10^{10.5}<L_{\mathrm{IR}}<\right.$ $10^{12}$ ), quite similar to the "average" IRAS galaxies;

iv) a high proportion of star forming objects, though with moderate SF rates (around ten solar masses per year), and a very small fraction of active galactic nuclei.

The importance of this "quiet" population of IR galaxies was clearly underestimated in the analysis of the IRAS survey and has to be taken into account in the calculations of the far-IR background. It is now clear that the far-IR background cannot be reproduced only by high- $z$ galaxies (Lagache et al. 2003), but that closer and colder galaxies are contributing also. Even if their contribution is probably rather low, they will form an important foreground for future infrared deep field observations. The relative contributions of nearby galaxies and of distant objects in the far-IR selected samples in particular, and in the far-IR background in general, will be estimated more precisely when we have completed the survey of fainter objects in the FSM and in the northern fields of the FIRBACK survey.

\section{References}

Baldwin, J. A., Phillips, M. M., \& Terlevich, R. 1981, PASP, 93, 5 Bertin, E., Dennefeld, M., \& Moshir, M. 1997, A\&A, 323, 685 Chapman, S. C., Smail, I., Ivison, R. J., et al. 2002, ApJ, 573, 66 Devriendt, J. E. G., Guiderdoni, B., \& Sadat, R. 1999, A\&A, 350, 381
Dessauge-Zavadsky, M., Pindao, M., Maeder, A., \& Kunth, D. 2000, A\&A, 355, 89

Dole, H., Gispert, R., Lagache, G., et al. (FIRBACK III) 2001, A\&A, 372,364

Elbaz, D., Cesarsky, C., Chanial, P., et al. 2002, A\&A, 384, 848

Glazebrook, K., Blake, C., Economou, F., Lilly, S., \& Colless, M. 1999, MNRAS, 306, $843 \mathrm{~S}$

Gruppioni, C., Ciliegi, P., Rowan-Robinson, M., et al. 1999, MNRAS, 305, 297

Hamuy, M., Walker, A. R., Suntzeff, N. B., et al. 1992, PASP, 104, 533

Hamuy, M., Suntzeff, N. B., Heathcote, S. R., et al. 1994, PASP, 106, 566

Helou, G., Khan, I. R., Malek, L., \& Boehmer, L. 1988, ApJS, 68, 151

Hopkins, A. M., Miller, C. J., Connolly, A. J., et al. 2002, AJ, 123, 1086

Hopkins, A. M., Afonso, J., Chan, B., et al. 2003, AJ, 125, 465

Howarth, I. D. 1983, MNRAS, 302, 301

Kennicutt, R. C. 1983, ApJ, 272, 54

Kennicutt, R. C. 1992, ApJ, 388, 310

Kessler, M. F., Steinz, J. A., Anderegg, M. E., et al. 1996, A\&A, 315, 27

Kim, D.-C., Sanders, D. B., Veilleux, S., Mazarella, J. M., \& Soifer, B. T. 1995, ApJ, 98, 129

Lagache, G., Dole, H., \& Puget, J. L. 2003, MNRAS, 338, 555

Lemke, D., Klaas, U., Abolins, J., et al. 1996, A\&A, 315, 64

Lutz, D., Spoon, H. W. W., Rigopoulou, D., Moorwood, A. F. M., \& Genzel, R. 1998, ApJ, 505, L103

Maffei, B. 1994, Ph.D. Thesis, Université Paris VII

Menzel, D. H. 1969, ApJS, 18, 221

Miller, L. H. 1956, J. Am. Statist. Ass., 47, 425

Osterbrock, D. E. 1989, Astrophysics of gaseous nebulae and active galactic nuclei (University Science Books)

Puget, J.-L., Lagache, G., Clements, D., et al. (FIRBACK I) 1999, A\&A, 345, 29

Sanders, D. B., \& Mirabel, F. 1996, ARA\&A, 34, 749

Sajina, A., Borys, C., Chapman, S., et al. 2003, MNRAS, 343, 1365

Sault, R. J., Teuben, P. J., \& Wright, M. C. H. 1995, A retrospective view of Miriad, in Astronomical Data Analysis Software and Systems IV, ed. R. Shaw, H. E. Payne, \& J. J. E. Hayes, ASP Conf. Ser., 77, 433

Scott, D., Lagache, G., Borys, C., et al. 2000, A\&A, 357, L5

Seaton 1979, MNRAS, 187, 73P

Soifer, B. T., Sanders, D. B., Madore, B. F., et al. 1987, ApJ, 320, 238

Stickel, M., Lemke, D., Klaas, U., et al. 2000, A\&A, 359, 865

Sullivan, M., Treyer, M., Ellis, R., et al. 2000, MNRAS, 312, 442

Tresse, L., Rola, C., Hammer, F., et al. 1996, MNRAS, 281, 847

Veilleux, S., \& Osterbrock, D. E. 1987, ApJ, 63, 295

Veilleux, S., Kim, D.-C., Sanders, D. B., Mazarella, J. M., \& Soifer, B. T. 1995, ApJ, 98, 171

Veilleux, S., Kim, D.-C., \& Sanders, D. B. 1999, AJ, 522, 113 